\title{
THE LBT BOÖTES FIELD SURVEY. I. THE REST-FRAME ULTRAVIOLET AND NEAR-INFRARED LUMINOSITY FUNCTIONS AND CLUSTERING OF BRIGHT LYMAN BREAK GALAXIES AT $Z \sim 3^{*}$
}

\author{
Fuyan Bian ${ }^{1}$, Xiaohui Fan ${ }^{1}$, Linhua Jiang ${ }^{1}$, IAN McGreer ${ }^{1}$, Arjun Dey ${ }^{2}$, Richard F. Green ${ }^{3}$, \\ Roberto Maiolino ${ }^{4}$, Fabian Walter $^{5}$, Kyoung-Soo LeE $^{6}$, and Romeel Davé ${ }^{1}$ \\ ${ }^{1}$ Steward Observatory, University of Arizona, 933 North Cherry Avenue, Tucson, AZ 85721, USA \\ ${ }^{2}$ National Optical Astronomy Observatory, 950 North Cherry Avenue, Tucson, AZ 85719, USA \\ ${ }^{3}$ Large Binocular Telescope Observatory and Steward Observatory, University of Arizona, 933 North Cherry Avenue, Tucson, AZ 85721, USA \\ ${ }^{4}$ Cavendish Laboratory, University of Cambridge, 19 J. J. Thomson Ave., Cambridge CB3 OHE, UK \\ ${ }^{5}$ Max-Planck-Institut für Astronomie, Königstuhl 17, D-69117 Heidelberg, Germany \\ ${ }^{6}$ Department of Physics, Purdue University, West Lafayette, IN 47906, USA \\ Received 2012 October 30; accepted 2013 June 17; published 2013 August 13
}

\begin{abstract}
We present a deep LBT/LBC $U_{\text {spec }}$-band imaging survey $\left(9 \mathrm{deg}^{2}\right)$ covering the NOAO Boötes field. A total of 14,485 Lyman break galaxies (LBGs) at $z \sim 3$ are selected, which are used to measure the rest-frame UV luminosity function (LF). The large sample size and survey area reduce the LF uncertainties due to Poisson statistics and cosmic variance by $\geqslant 3$ compared to previous studies. At the bright end, the LF shows excess power compared to the best-fit Schechter function, which can be attributed to the contribution of $z \sim 3$ quasars. We compute the rest-frame near-infrared LF and stellar mass function (SMF) of $z \sim 3$ LBGs based on the $R$-band and [4.5 $\mu \mathrm{m}$ ]-band flux relation. We investigate the evolution of the UV LFs and SMFs between $z \sim 7$ and $z \sim 3$, which supports a rising star formation history in the LBGs. We study the spatial correlation function of two bright LBG samples and estimate their average host halo mass. We find a tight relation between the host halo mass and the galaxy star formation rate (SFR), which follows the trend predicted by the baryonic accretion rate onto the halo, suggesting that the star formation in LBGs is fueled by baryonic accretion through the cosmic web. By comparing the SFRs with the total baryonic accretion rates, we find that cosmic star formation efficiency is about 5\%-20\% and it does not evolve significantly with redshift, halo mass, or galaxy luminosity.
\end{abstract}

Key words: galaxies: evolution - galaxies: formation - galaxies: high-redshift

Online-only material: color figures

\section{INTRODUCTION}

The redshifts between $1<z<3$ were the most active epochs of galaxy formation, when the star formation rate (SFR) density and the activity of bright quasars reached their peaks (e.g., Madau et al. 1996; Fan et al. 2001). In this epoch, the Hubble sequence observed in the nearby universe was being built up and about $50 \%$ of the present day stars formed (Dickinson et al. 2003). Therefore, observations in this redshift range provide crucial clues for understanding the formation and evolution of galaxies. The Lyman break technique has been well developed for surveying galaxies in this redshift range (e.g., Steidel et al. 1996a, 1996b). Large samples of high-redshift star-forming galaxies have been established with this method (e.g., Steidel et al. 2003).

Using samples of Lyman break galaxies (LBGs), the restframe UV luminosity functions (LFs) from $z \sim 2$ to $z \sim 7-8$ have been well studied. (e.g., Ly et al. 2009; Reddy \& Steidel 2009; Steidel et al. 1999; Sawicki \& Thompson 2006a; Bouwens et al. 2007, 2008; Yan et al. 2011). The rest-frame UV LF is a fundamental tracer of galaxy formation and evolution; it

\footnotetext{
* Based on data acquired using the Large Binocular Telescope (LBT). The LBT is an international collaboration among institutions in the United States, Italy, and Germany. LBT Corporation partners are: The University of Arizona on behalf of the Arizona university system; Istituto Nazionale di Astrofisica, Italy; LBT Beteiligungsgesellschaft, Germany, representing the Max-Planck Society, the Astrophysical Institute Potsdam, and Heidelberg University; The Ohio State University, and The Research Corporation, on behalf of The University of Notre Dame, University of Minnesota, and University of Virginia.
}

is used to compute the UV luminosity density by applying a dust extinction correction and to constrain the history of star formation (e.g., Madau et al. 1996).

However, LF measurement remains uncertain for $z \sim 2-3$ LBGs, the measured faint-end slope $(\alpha)$ of the Schechter function ranges from the shallowest with $\alpha=-1.05$ to the steepest with $\alpha=-1.88$ (e.g., Ly et al. 2009; Reddy et al. 2008); at the bright end, there are discrepancies between Steidel et al. (1999) and Le Fèvre et al. (2005) for the galaxies at $z \sim 3$ by factors $1.6-6.2$, and $z \sim 4$ by factors $2-3.5$. In addition, the evolution of the bright-end UV LF of high-redshift LBGs is still not well constrained. Sawicki \& Thompson (2006b) claimed that the number density of bright LBGs decreases with redshifts, while Bouwens et al. (2007) found that the number density remains constant. Furthermore, most of the small area surveys lack information on the most luminous LBGs, i.e., with $M_{1700 \AA}<-23$, due to the small surface density of these luminous LBGs.

Galaxy clustering can be used to test the hierarchical theory of structure formation, which predicts that the clustering of dark matter halos strongly depends on their masses and assembly history (e.g., Mo \& White 1996). Numerical simulations can predict the dark matter distribution given the underlying cosmology and the initial matter power spectrum derived from the cosmic microwave background measurements (e.g., Spergel et al. 2007). Distributions of galaxies and dark matter are connected by the halo occupation distribution (HOD; e.g., Zheng et al. 2007). The mass of dark matter halos can be determined with HOD models. Many studies have shown that LBGs are 
strongly clustered (e.g., Adelberger et al. 1998; Giavalisco et al. 1998), and the brighter galaxies are more strongly clustered at large scales (e.g., Adelberger et al. 2005; Ouchi et al. 2005; Lee et al. 2006; Hildebrandt et al. 2007).

In addition, the correlation function of LBGs shows excess power at small scales $\left(\theta<1^{\prime \prime}\right)$, implying multiple galaxies within the same massive dark matter halo in the context of HOD (Ouchi et al. 2005; Lee et al. 2006, 2009). Combined with the UV LF, clustering results also can be used to infer the nature of star formation in the LBGs and its dependence on their host halo mass (Lee et al. 2009).

In previous deep field surveys, survey areas were relatively small. The largest $z \sim 3 \mathrm{LBG}$ surveys so far with spectroscopic redshifts are the Keck Baryonic Structure Survey (KBSS; Steidel et al. 2003, 2004) and the VLT LBG Redshift Survey (VLRS; Bielby et al. 2011). The KBSS and VLRS cover a total area of around $1 \mathrm{deg}^{2}$ and $3 \mathrm{deg}^{2}$, respectively, with $\approx 2000$ spectroscopic redshifts (Reddy \& Steidel 2009; Bielby et al. 2013). The largest coherent structures revealed in these surveys have sizes comparable to the field size: we clearly have not reached the scale of the largest structures at that time. The small sample size means that only simple statistics can be computed, and it is difficult to subdivide the sample to probe the dependence of clustering on the intrinsic properties of the galaxies. Given the difficulty in obtaining even larger spectroscopic samples of faint LBGs, the only effective way to expand the sample size by a large factor is through photometrically selected samples. For example, the Garching-Bonn deep survey (Hildebrandt et al. 2007) covers $\approx 2 \mathrm{deg}^{2}$, and $\approx 8000 z \sim 3$ photometrically selected LBGs are selected to study the clustering properties.

The key to establishing a large $z \sim 3$ LBG sample is the availability of deep multi-wavelength imaging, especially deep $U$-band imaging. The Large Binocular Camera (LBC; Giallongo et al. 2008) — blue on the left arm ("DX-side") of the $2 \times 8.4 \mathrm{~m}$ Large Binocular Telescope (LBT) is specially designed to have high throughput and good image quality in the blue. We have carried out a large LBC survey of the NOAO Deep Wide Field Survey (NDWFS; Jannuzi \& Dey 1999) Boötes field (9 $\left.\mathrm{deg}^{2}\right)$ in the $U_{\text {spec }}$ band $\left(\lambda_{0}=3590 \AA, F W H M=540 \AA\right)$ and $Y$ band $\left(\lambda_{0}=9840 \AA\right.$, FWHM $=420 \AA$; Figure 1$)$, building on the unique multi-wavelength data set already available for the Boötes field, while filling in two critical wavelength gaps. The survey area is about five times larger than previous studies (e.g., COSMOS; Scoville et al. 2007), which allows us to build a larger LBG sample to further study the LF and clustering properties, especially for the brightest LBGs at redshift $z \sim 3$.

This is the first in a series of papers. In this paper, we will focus on the photometrically selected LBGs and study their UV and NIR LF and clustering properties, especially for the bright LBGs. In following papers, we will focus on spectroscopic confirmation of the most luminous LBGs.

The paper is organized as follows. Observations are discussed in Section 2. Data reduction is described in Section 3. Sample selection is given in Section 4. We present our bright-end restframe UV and near-IR LF and stellar mass function (SMF) results in Sections 5 and 6 and discuss the evolution of UV LF and SMF with cosmic time in Section 7. Clustering results are presented in Section 8. Finally, we summarize our results. Throughout this paper, we use the following cosmological parameters for the calculations: Hubble constant, $H_{0}=70 \mathrm{~km} \mathrm{~s}^{-1} \mathrm{Mpc}^{-1}$; dark matter density, $\Omega_{\mathrm{M}}=0.30$; and dark energy density, $\Omega_{\Lambda}=0.70$ for a flat universe (e.g., Spergel et al. 2007). All the magnitudes are expressed in the AB magnitude system (Oke \& Gunn 1983).

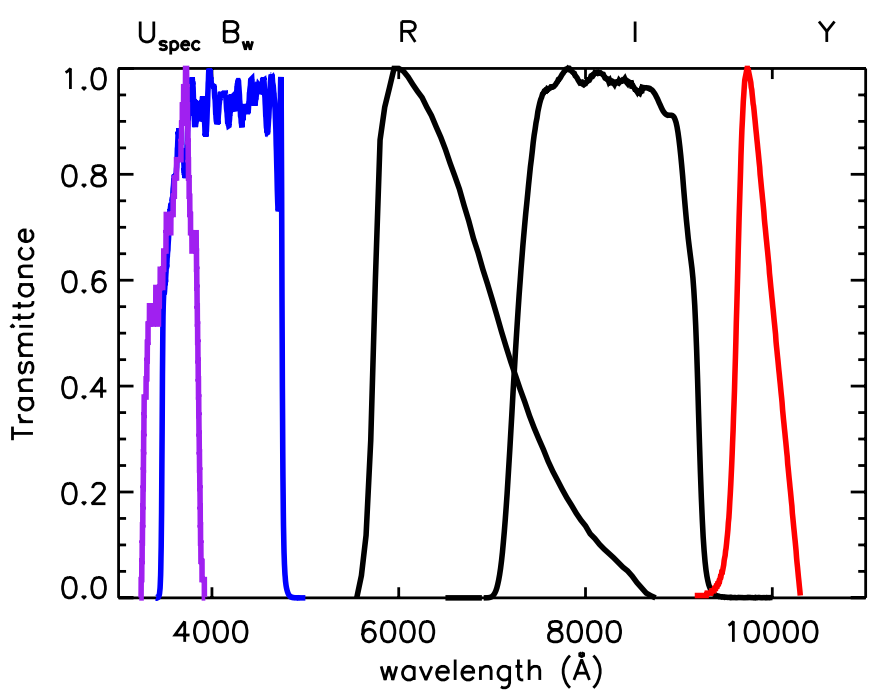

Figure 1. Relative transmission curves of the LBC $U_{\text {spec-band (purple curve) }}$

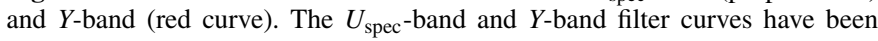
corrected by both the CCD Q.E. curve and the atmosphere transmission. This plot also shows the transmission curves of the $B \mathrm{w}$ (blue curve), $R$ and $I$ bands in the NDWFS Boötes field. All the transmission curves are normalized by the peak transmittance for clarity.

(A color version of this figure is available in the online journal.)

\section{OBSERVATIONS}

The Boötes field $\left(\alpha(J 2000)=14^{\mathrm{h}} 32^{\mathrm{m}}, \delta(J 2000)=\right.$ $\left.+34^{\circ} 16^{\prime}\right)$, one of the NDWFS fields, is a $9 \mathrm{deg}^{2}$ field covered by a deep multicolor survey in $B \mathrm{w}, R$, and $I$ optical broad bands (Jannuzi \& Dey 1999), and $J, H$, and $K s$ near-infrared (nearIR) bands (Elston et al. 2006; Gonzalez et al. 2010). A shallow $z$-band survey was carried out by Cool (2007). Additionally, this deep and wide field has been observed at other wavelengths, including in the X-ray with Chandra (Murray et al. 2005b; Kenter et al. 2005; Brand et al. 2006), UV with Galaxy Evolution Explorer (GALEX; Hoopes et al. 2003), infrared with Spitzer IRAC (Ashby et al. 2009) and MIPS (Soifer \& Spitzer/NOAO Team 2004), and radio with the Very Large Array (Becker et al. 1995; de Vries et al. 2002). A redshift survey (the AGN and Galaxy Evolution Survey; Kochanek et al. 2012) has also been conducted on this field, with spectra of roughly 17,000 galaxies and 3000 active galactic nuclei (AGNs) down to $I \approx 20$ using the Hectospec instrument mounted on the $6.5 \mathrm{~m} \mathrm{MMT}$.

There are two significant gaps in the optical wavelength coverage in this field: one is between the $B \mathrm{w}$ broad band and GALEX NUV, and the other is between $I$ band and $J$ band. To fill these two gaps, we have carried out the LBT Boötes field survey with the LBCs mounted on the $2 \times 8.4 \mathrm{~m}$ LBT in binocular mode with $U_{\text {spec }}$-band $\left(\lambda_{0}=3590 \AA, \mathrm{FWHM}=540 \AA\right)$ and $Y$-band $\left(\lambda_{0}=9840 \AA, F W H M=420 \AA\right)$ imaging (Figure 1$)$. The LBCs are two wide-field cameras, and each is mounted on one of the LBT prime foci. These two cameras can observe the same sky field simultaneously. The blue channel is optimized for the UV-B bands, and the red channel is optimized for the VRIz bands. The CCD quantum efficiencies are $\approx 50 \%$ and $\approx 10 \%$ in the $U_{\text {spec }}$ and $Y$ bands, respectively. The CCD pixel size is $0^{\prime \prime} .225$ pixel $^{-1}$. Each of the cameras consists of four $2 \mathrm{k} \times$ $4 \mathrm{k}$ chips, resulting in a $23 \times 23 \operatorname{arcmin}^{2}$ field of view (FoV). Because the layout of the CCD is not a square, the total effective FoV is about $470 \operatorname{arcmin}^{2}$.

The primary goal of our survey is to use the unique $Y$ - and $U_{\text {spec }}$-band data to search for $z \sim 7$ quasars at the epoch of 


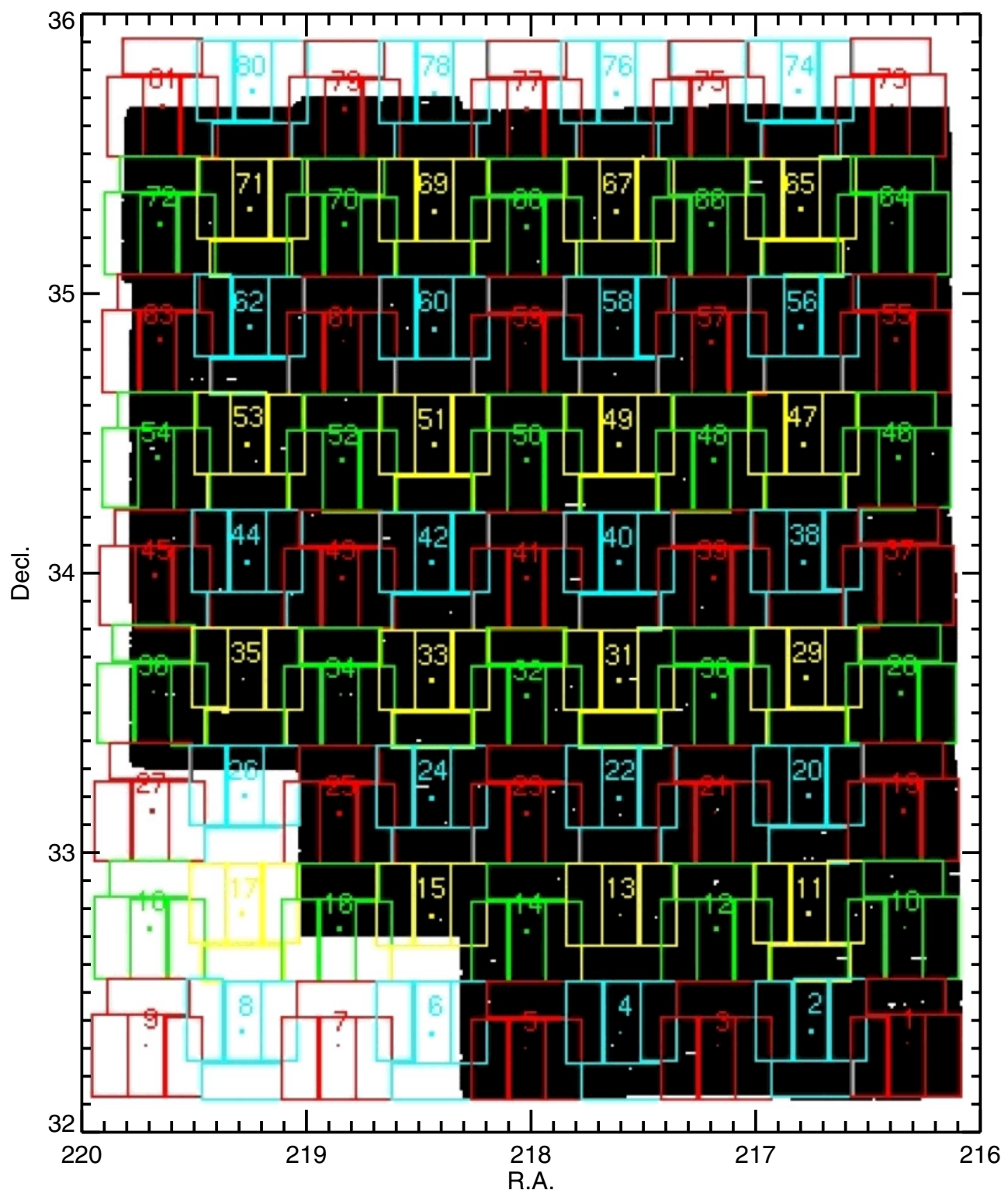

Figure 2. $U_{\text {spec }}$ coverage map for the Boötes field. The black region is covered by the NDWFS $B \mathrm{w}, R$, and $I$ bands. There are a total of 81 fields designed to cover the whole rectangular region, 63 of which have overlaps with the NDWFS broadband coverage.

(A color version of this figure is available in the online journal.)

cosmic reionization and $z \sim 3 \mathrm{LBGs}$ at the epoch of the peak in star-forming and quasar activity. In this paper, we focus on the $U_{\text {spec }}$-band data to establish an LBG sample and publish the scientific results of the LBG sample.

A total of 81 pointings were designed to cover the entire rectangular region (Figure 2), and 63 of them, which overlap with the NDWFS optical band coverage by more than $50 \%$ of the LBC FoV, were observed. The total survey area is about $8.8 \mathrm{deg}^{2}$. For each individual field, a $1200 \mathrm{~s}$ exposure time observing block is designed. The total $1200 \mathrm{~s}$ exposure time is divided into five individual $240 \mathrm{~s}$ exposures with $30^{\prime \prime}$ dither patterns. The dither pattern allows us to fill up the chip gaps, remove cosmic rays and bad pixels, and reduce the effects of the bright stars. The position angles of the neighboring fields in the declination direction have a $180 \mathrm{deg}$ difference and an $\approx 4^{\prime} \times 7^{\prime}$ overlap region, which allows us to compare the calibration with different position angles.

The observations were carried out in dark time from 2008 January to 2009 March in queue observing mode. There are a total of $718 U_{\text {spec }}$-band science images obtained with a total open shutter time of $\approx 47.8 \mathrm{hr}$. About $30 \%$ of the data (222 images) are unusable, in which $20 \%$ (151 images) are trailed due to motion of the telescope during the exposure and $10 \%$ (71 images) have poor image quality (FWHM $>1$ 1".8). The images with FWHM $>1$ 1".8 do not make a significant contribution (less than $50 \%$ compared to images with median FWHM) to the depth of the final co-added images (see details in Section 3.4).

The median air mass is 1.11 and the median FWHM is $1^{\prime \prime} .25$ (1".33) after (before) removing the bad quality images. The final average effective exposure time for each individual field is about 30 minutes.

\section{DATA REDUCTION}

\subsection{Bias and Flat Correction}

All images are inspected visually to remove elongated images due to tracking and guiding issues during observing before further processing. About $20 \%$ of images are removed in this step. 


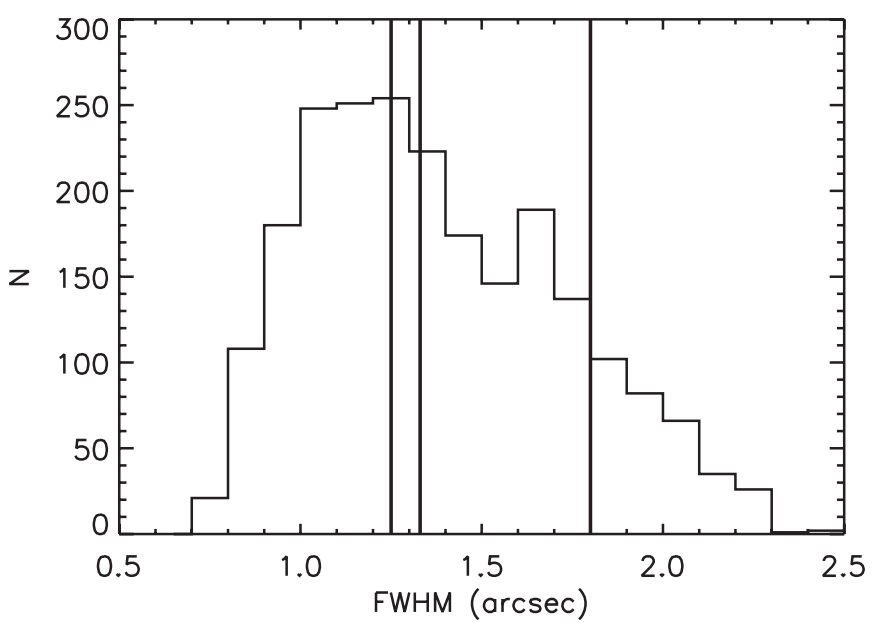

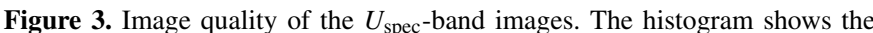
distribution of the FWHMs of each chip of the LBC. The median of the FWHM is $1^{\prime \prime} .33 / 1^{\prime \prime} .25$ before/after removing the bad image quality images (FWHM > 1". 8 ). The three vertical lines mark the FWHMs of $1^{\prime \prime} .25,1^{\prime \prime} .33$, and $1^{\prime \prime} 8$ from left to right.

Data are reduced using custom IDL routines. The bias level measured from the overscan region is subtracted from individual bias frame images, which are then combined to construct the master bias frame. Super-sky flats are created by combining the science frames in each observation run, scaling by the mean value with $3 \sigma$ clipping to remove cosmic rays and objects. Science images are overscan and bias subtracted, and then divided by the super-sky flats to correct the CCD response. Finally, cosmic rays in the images were identified rejected using an identification algorithm based on Laplacian edge detection (van Dokkum 2001).

\subsection{Image Quality Measurement}

We estimate the FWHM of each scientific frame as follows: (1) a few stars across the field are selected, and the FWHM of the stars is measured using imexamine in $\mathrm{IRAF}^{7}$ as an input for the next step; (2) a catalog for each field is created by SExtractor (Bertin \& Arnouts 1996) with the parameter of SEEING_FWHM set to the value from step 1. All objects with six or more connected pixels with flux 3.0 times greater than the sky noise are detected. Well detected bright stars $\left(18.5<\mathrm{mag}_{\text {auto }}<20.0\right.$ and star_class $\left.>0.95\right)$ are selected from the SExtractor output catalog; and (3) image coordinates of well detected bright stars are used as the input information for the FWHM measure task, psfmeasure, in IRAF. This task fits the bright stars profile as a Moffat distribution function. The median of FWHMs of the best-fit Moffat profile in each frame is used to represent its image quality.

Figure 3 shows the $U_{\text {spec }}$-band image quality. Frames with FWHM larger than 1".80 were not used for further analysis and co-addition. After removing the bad image quality images (FWHM > 1".8), the median FWHM is 1".25, and the first and third quartiles are 1".07 and 1".50, respectively.

\subsection{Astrometric Calibration}

The catalogs created by SExtractor in the previous section are used as an input catalog for Software for Calibrating AstroMetry and Photometry (SCAMP; Bertin 2006). To compute

\footnotetext{
7 IRAF is distributed by the National Optical Astronomy Observatory, which is operated by the Association of Universities for Research in Astronomy, Inc., under cooperative agreement with the National Science Foundation.
}

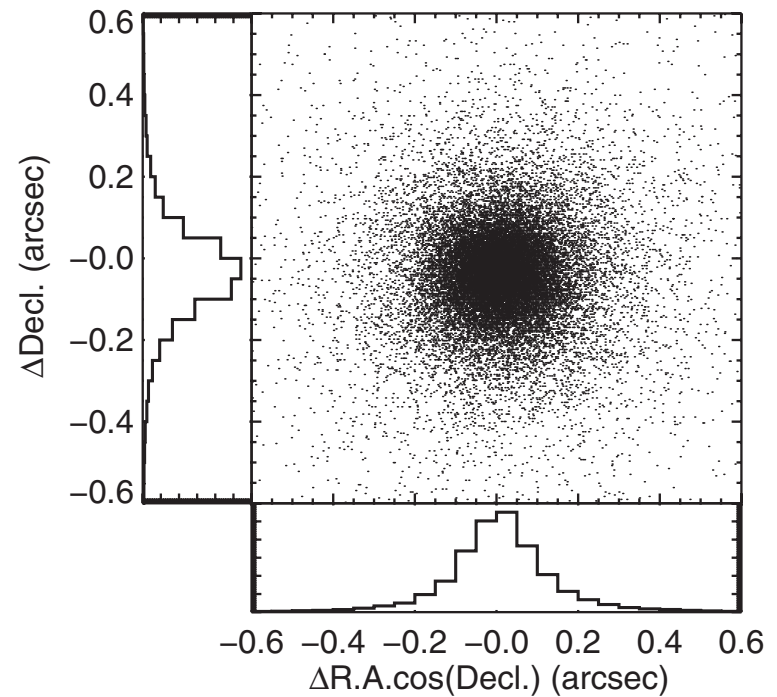

Figure 4. Accuracy of the astrometry. The distribution of the difference of R.A. $\times \cos ($ Decl. $)$ and Decl. between LBC $U_{\text {spec }}$-band images and SDSS images. The FWHMs of both distributions are about 0.2 , which corresponds to a $1 \sigma$ astrometric uncertainty of 0.08 .

the astrometric solution, we use well detected objects that meet the following criteria: (1) the object is not saturated; (2) the signal-to-noise ratio of the object is greater than 10; and (3) the FWHM of the object in the SExtractor output catalog is between $2^{\prime \prime}$ and $10^{\prime \prime}$. These well detected objects from the input catalog are used to search for matches in the SDSS-DR6 catalog within a $3^{\prime \prime}$ radius. We first use a linear model with only an $x, y$ directional offset and without rotations to obtain a rough astrometry solution. Based on this solution, we then use a third-order polynomial to fit the $x, y$ offset and the rotation to get a refined solution. Using this procedure, an accurate astrometric solution is derived for each field with the $1 \sigma$ uncertainty less than $0^{\prime \prime} 1$ in both R.A. and Decl. direction (Figure 4).

Previous studies (e.g., Cool 2007) have shown that there is $\approx 0$ '.3 offset between the NDWFS catalog ${ }^{8}$ astrometry and the Sloan Digital Sky Survey (SDSS) astrometry. Thus, we also register the NDWFS $B \mathrm{w}-, R$-, and $I$-band images to the SDSS-DR6 catalogs using the same method.

\subsection{Image Co-addition}

Before co-adding images, we subtract the sky background from the science frames and generate a weight map for each frame. The background is constructed from the -object image created by SExtractor, in which the detected objects have been subtracted from the image. First, the -object image is divided into $\approx 100$ background mesh regions with the size of 130 pixel $\times$ 130 pixel. Then the median background is computed for each region, and it is fitted with a second-order polynomial and subtracted from the science images. The weight map value is computed as follows:

$$
w=\frac{1}{\mathrm{FWHM}^{2} \sigma^{2}},
$$

where the FWHM is described in Section 3.2, and $\sigma^{2}$ is the sky variance.

SWarp (Bertin et al. 2002) is used to co-add images for each field. First, the input science images and weight maps

\footnotetext{
8 http://www.noao.edu/noao/noaodeep/DR3/dr3cats.html
} 


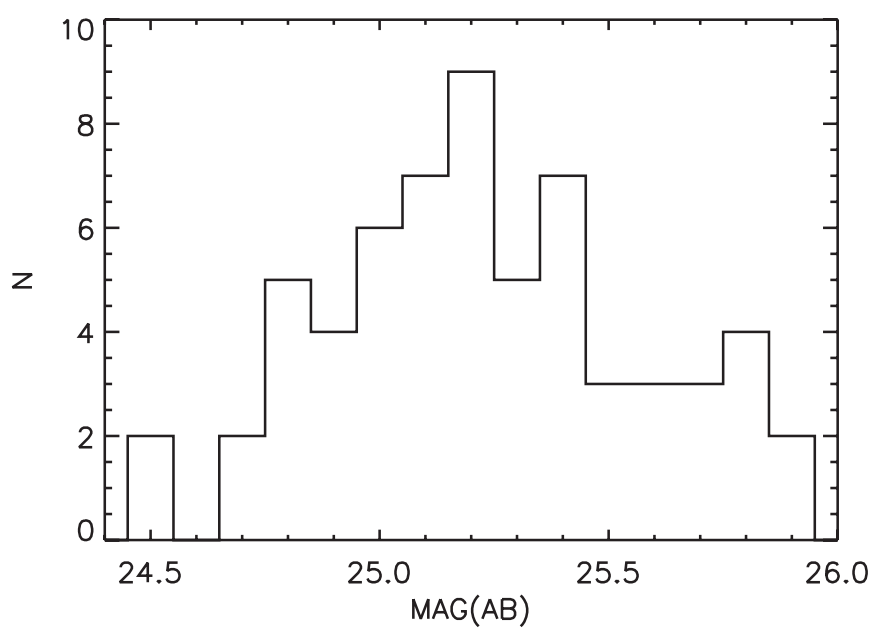

Figure 5. Distribution of magnitude with $5 \sigma$ detections for the $U_{\text {spec }}$ band. The median depth is 25.2 mag.

are resampled to a common pixel grid. The interpolation function we use to re-sample images is LANCZOS3, a $\prod_{d}$ $\operatorname{sinc}\left(\pi x_{d}\right) \operatorname{sinc}\left((\pi / 4) x_{d}\right)$ response function with $\left(-3<x_{d} \leqslant 3\right)$. The output co-added image is a weighted average of input values after $3 \sigma$ clipping:

$$
F=\frac{\sum_{i} w_{i} f_{i}}{\sum_{i} w_{i}},
$$

where $w_{i}$ is the weight of the pixel from resampled weighted map, and $f_{i}$ is flux value of the pixel from the science image. The output co-added weighted map is the sum of input weights:

$$
W=\sum_{i} w_{i} .
$$

Finally, we create exposure maps to record the exposure time for each pixel in the co-added images.

\subsection{Photometric Calibration}

The imaging data are calibrated with SDSS data release 6 (DR6) photometry. The SDSS $u$-band transmission curve is similar to LBC $U_{\text {spec }}$, with $\lambda_{0}=3540 \AA$ and FWHM $=570 \AA$. Bright stars $(18.0<u<19.5)$ in the NO. 57 field which was taken in photometric conditions are used to determine an offset between the LBC $U_{\text {spec }}$-band and SDSS $u$-band images, and the color term coefficient. We find the color term is very small, about $-0.01 \times(u-g)$. Bright stars (star_class $>0.9$ and magerr_aper $\left.\left(8^{\prime \prime}\right)<0.02\right)$ in the overlap regions are used to determine the offset of the photometric zero point in other fields. These stars are also used to check the magnitude difference between two neighboring fields. As the position angle of the neighboring frame is offset by $180 \mathrm{deg}$, this check will give us the upper limit of the magnitude uncertainty from the calibration. The average standard deviation of the difference is $\approx 0.05$, implying the photometric accuracy is $\approx 0.05$. Another way to check the photometric accuracy is to compare magnitudes of stars within the same field observed in different individual exposures; from this check, we find the standard deviation is about 0.04; therefore, the $U_{\text {spec }}$-band magnitude uncertainty in the Boötes survey is $\approx 0.04-0.05$. The magnitude uncertainty is mainly introduced by the flat field and the non-uniformity of the image quality across the field.
Table 1

Summary of the $U_{\text {spec }^{-}}, B$ w-, and $R$-band $5 \sigma$ Depth

\begin{tabular}{lcccc}
\hline \hline Band & $\begin{array}{c}\lambda_{0}{ }^{\mathrm{a}} \\
(\AA)\end{array}$ & $\begin{array}{r}\text { Median Image Quality } \\
(\operatorname{arcsec})\end{array}$ & $\begin{array}{c}\text { Exposure Time } \\
(\mathrm{s})\end{array}$ & Depth $^{\mathrm{b}}$ \\
\hline$U_{\text {spec }}$ & 3590 & 1.25 & 1920 & 25.2 \\
$B \mathrm{~W}$ & 4133 & 1.10 & 8400 & 26.3 \\
$R$ & 6407 & 1.10 & 6000 & 25.3 \\
\hline
\end{tabular}

Notes.

${ }^{\text {a }}$ Effective wavelength

${ }^{\mathrm{b}}$ Depth for $5 \sigma$ detection in a $2 \times$ FWHM aperture.

\subsection{Survey Depth}

We use the following process to determine the $5 \sigma$ detection depth in the $U_{\text {spec }}$-band image for each field: (1) we generate five catalogs for each individual field, and each catalog has 6000 simulated stars with magnitudes between 22.0 and 28.0. The Moffat profile with the FWHM value the same as the image quality of each individual field is used to generate the light profile of stars, and these fake stars are added to the real $U_{\text {spec-band images; and (2) we use the SExtractor to detect }}$ and measure the simulated stars in the same manner as for the primary catalogs. The standard deviations between the measured magnitude and the input magnitude are calculated in different magnitude bins. For each field, the magnitude bin in which the standard deviation is 0.2 is considered as the magnitude limit with $5 \sigma$ detection. The median depth is around 25.2 AB magnitude, and the first and third quartiles are 25.0 and 25.4 AB magnitude, respectively. The distribution of the $5 \sigma$ limit magnitude is shown in Figure 5.

In this paper, we also use the $B_{\mathrm{W}}, R$ optical broadband images $^{9}$ taken by the NOAO/KPNO Mosaic-1 $(8 \mathrm{~K} \times 8 \mathrm{~K}$ CCD) Wide Field Imager on $4 \mathrm{~m}$ Mayall Telescope at Kitt Peak National Observatory (KPNO) covering the entire field. The typical exposure times in these two bands are $\approx 8400 \mathrm{~s}$ and $\approx 6000 \mathrm{~s}$, respectively. The median image qualities of both $B_{\mathrm{W}}-$ and $R$-band images are 1.11. The median $5 \sigma$ depths are 26.3 and 25.3 AB magnitude in $B_{\mathrm{W}}$ and $R$ bands, respectively (see Table 1). The median image qualities and $5 \sigma$ depths of $B_{\mathrm{W}^{-}}$and $R$-band images are adopted from the third data release of the NDWFS. ${ }^{10}$

\section{LBG SAMPLE SELECTION}

\subsection{Photometry}

To select LBGs, the crucial step is to find the $U$-dropout objects. In some cases, the $U$-dropout objects cannot be directly detected and measured on $U$-band images, therefore, the NDWFS $R$-band images are used as the detection images in this study.

First, we map the NDWFS $R$-band images using SWarp to the LBT $U_{\text {spec }}$-band images with the same pixel size and image size. Then we use SExtractor in double-image mode with the mapped $R$-band images as detection images and $U_{\text {spec }}$-band images as measurement images. A source is considered to be detected if the number of connected pixels with flux 0.6 times greater than the sky $\sigma$ exceeds four pixels after the original image is convolved with a $9 \times 9$ convolution mask of a Gaussian point-spread function with FWHM $=5.0$ pixels in the $R$-band

\footnotetext{
9 ftp://archive.noao.edu/ndwfs/dr3/

10 http://www.noao.edu/noao/noaodeep/DR3/dr3-data.html
} 

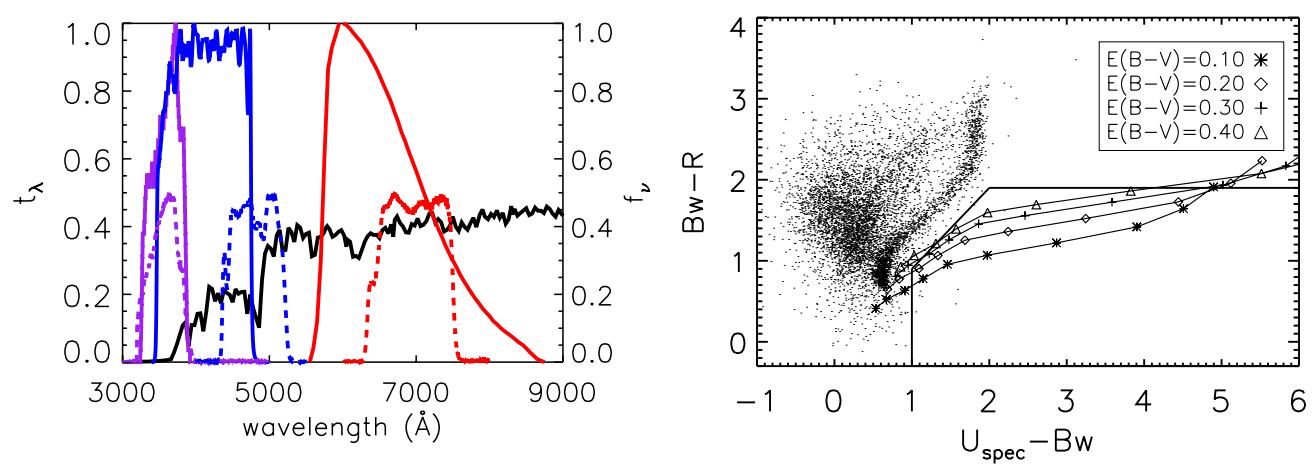

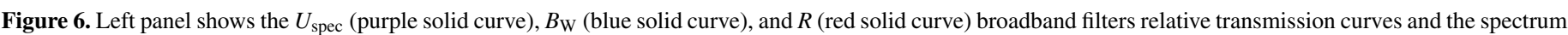

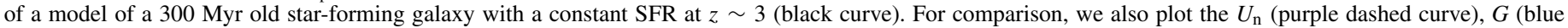

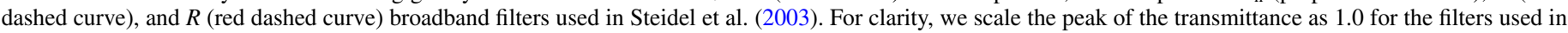

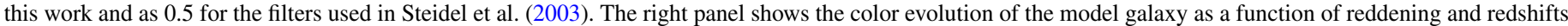

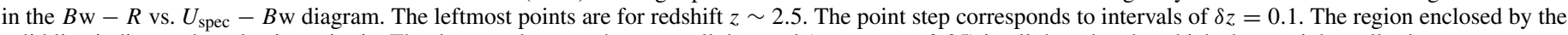
solid line indicates the selection criteria. The dots are the stars that are well detected (mag_err $<0.05)$ in all three bands, which show a tight stellar locus.

(A color version of this figure is available in the online journal.)

image. We reduce the NDWFS $B_{\mathrm{W}}$ data in the same manner. The astrometry has been registered to the SDSS-DR6 catalog, as discussed in Section 3.3.

The $U_{\text {spec }}$-band exposure map masks are used as the external flags to obtain the exposure time for each object. Objects with $U_{\text {spec }}$-band exposure times less than $720 \mathrm{~s}$ are ignored. The aperture magnitude (mag_aper) with aperture size of $2.0 \times$ FWHM is then used for color selection. The aperture correction is estimated for each field by applying an $8^{\prime \prime}$ aperture to measure the total flux of bright stars. Then the aperture corrections (i.e., $\Delta$ mag $=$ mag_aper $\left(8^{\prime \prime}\right)$-mag_aper $(2 \times$ FWHM $\left.)\right)$-with values around -0.2 - are used to correct the flux loss due to measurement in relatively small apertures. For those $U_{\text {spec }}$ non-detected sources, we set the magnitude upper limits at $1 \sigma$. Sources from the $U_{\text {spec }}, B_{\mathrm{W}}$ and $R$-band catalogs within $1^{\prime \prime}$ positional variation are matched together to generate the $U_{\text {spec }}-B_{\mathrm{W}}$ versus $B_{\mathrm{W}}-R$ color-color diagram.

\subsection{Sample Selection Criteria}

To determine the LBG sample selection, the BC03 standard stellar synthesis population model (Bruzual \& Charlot 2003) is used to build a series of spectral templates of star-forming galaxies. We adopt a spectral model with a constant SFR, a Salpeter initial mass function (IMF; Salpeter 1955), one solar metallicity abundance, and an age of $300 \mathrm{Myr}$ to simulate the spectra of star-forming galaxies. This model will give us a typical intrinsic LBG spectral energy distribution (SED; e.g., Steidel et al. 2003).

The templates are modified by the intergalactic medium (IGM) absorption model of Madau (1995) and reddened using the attenuation law of Calzetti et al. (2000) with reddening of $E(B-V)=0.0-0.4$. Then the wavelength of the spectra is shifted by a factor of $1+z$ to derive the spectra in the redshift range from 2.5 to 3.6. Figure 6 shows one of the star-forming galaxy template spectra with $E(B-V)=0.2$ at a redshift of $z=3$, and how the galaxies with given $E(B-V)$ evolve in the $U_{\text {spec }}-B_{\mathrm{W}}$ versus $B_{\mathrm{W}}-R$ color-color diagram with redshift. The leftmost point for each track represents a redshift of $z=2.5$ and each step corresponds to a redshift interval of 0.1 . The lower redshift limit is primarily determined by the $U_{\text {spec }}-B_{\mathrm{W}}$ cut, and the upper redshift limit is mainly determined by the $B_{\mathrm{W}}-R$ cut. We also plot the well detected stars (mag_err $<0.05)$ in all three bands in the color-color diagram. It shows a stellar locus, which is well separated from the tracks of the $z \sim 3$ LBGs. To select the LBGs with $2.7<z<3.3$ and maintain enough separation from the stellar locus to reduce the contamination rate from stars, the following selection criteria for $z \sim 3 \mathrm{LBGs}$ are adopted:

$$
\begin{array}{r}
U_{\text {spec }}-B \mathrm{w}>1.0, \\
B \mathrm{w}-R<1.9, \\
B \mathrm{w}-R<U_{\text {spec }}-B \mathrm{w}+0.1, \\
R<25.0 .
\end{array}
$$

\subsection{Selection Function}

For a well detected galaxy with intrinsic $U_{\text {spec }}-B_{\mathrm{W}}$ and $B-R$ colors right in the color-color selection region, the probability of selecting this galaxy as an LBG is mainly influenced by the range of intrinsic SEDs of the population at that redshift, some of which could scatter the observed colors beyond the selection region boundaries or band detection limits. In this paper, the completeness is derived from the selection function, which describes the detection probability $(P(m, z$, SED $))$ of an LBG spectral template with a given redshift, magnitude, and SED falling within the selection criteria.

The procedures to calculate the LBG selection function are as follows: the spectral templates generated in Section 4.2 are used to derive the intrinsic color distribution. For galaxies with a constant SFR, Salpeter IMF, solar metallicity, and $300 \mathrm{Myr}$ age, as is typical for an LBG SED (e.g., Steidel et al. 2003), the SEDs of the galaxies are influenced only by the reddening $E(B-V)$. Therefore, in this case, $P(m, z, \mathrm{SED})$ is equivalent to $P(m, z, E(B-V))$. The detection probability is then a function of magnitude, redshift, and dust extinction. The $E(B-V)$ distribution of our sample is taken from the results of Reddy et al. (2008), Table 5, which is from -0.1 to 0.4 . A series of SEDs are generated with $2.0<z<4.0(\Delta z=0.1)$ and $E(B-V)$ from -0.1 to $0.4(\Delta E(B-V)=0.1)$. The broadband colors are fixed for a given magnitude, dust extinction and redshift combination. The expected colors of $U_{\text {spec }}-B_{\mathrm{W}}$ and $B_{\mathrm{W}}-R$ for a given redshift and $E(B-V)$ are derived by convolving the spectral template with the filter curves. Ten thousand simulated objects, following expected $U_{\text {spec }}-B_{\mathrm{W}}$ and $B_{\mathrm{W}}-R$ colors, are put on the $U_{\text {spec }}, B_{W}$, and $R$ images for each $R$ magnitude $(\Delta \mathrm{mag}=0.5)$, redshift $(\Delta z=0.1)$, and $E(B-V)(\Delta E(B-V)=0.1)$ bin, based on their expected noise characteristics on the real images. 


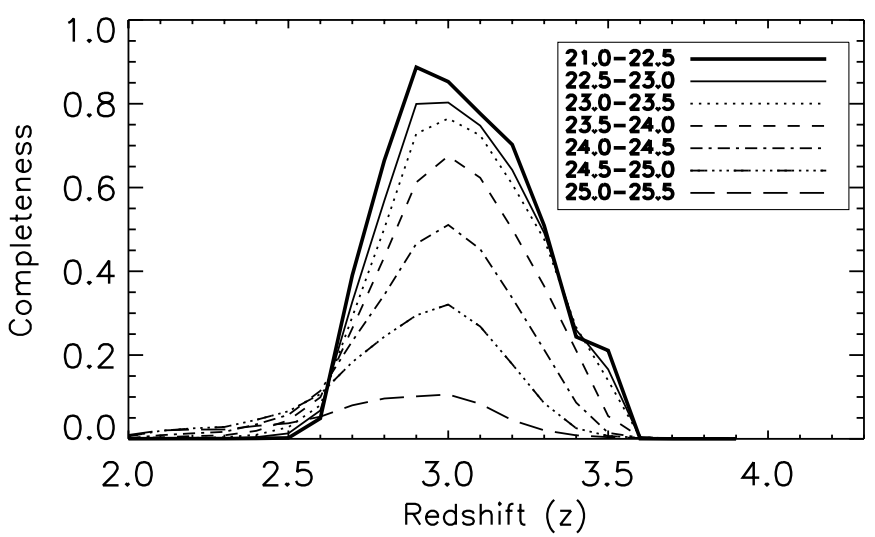

Figure 7. Selection completeness as a function of redshift in different $R$-band magnitude bins.

The size of faint LBGs is compact with $r_{e}<0$ '.5 (e.g., Ferguson et al. 2004), which cannot be resolved in our ground-based images, therefore, we use the Moffat profile to simulate the light distribution of the LBGs. The method of detection and measurement of these simulated objects is the same as that used for our real objects. Then the $P(m, z, E(B-V))$ is derived from the fraction of the simulated objects meeting the selection criteria. By weighting $P(m, z, E(B-V))$ with the distribution of the $E(B-V)$ (Reddy et al. 2008), the selection function, i.e., the LBG detection probability, as a function of redshift for a given magnitude bin is finally determined.

Figure 7 shows the selection function as a function of redshift in different $R$-band magnitude bins from 21.0 to 25.0. The redshift range is $2.7<z<3.3$ with a peak at $z=2.9$. The completeness decreases for fainter galaxies, because the magnitude error scatters color-color points out of the selection region and the detection completeness for $R$ band drops very quickly for the faint end (e.g., $R=24.5-25.0$ ). For the fainter magnitude bins, low-redshift galaxies have a greater chance to be scattered into the selection region due to the large magnitude errors. Thus the redshift distribution of faint galaxies shows an extended tail at the low redshift end.

\subsection{A Sample of Photometrically Selected $z \sim 3$ LBGs}

Figure 8 shows the distribution of objects from the Boötes field in the $U_{\text {spec }}-B_{\mathrm{W}}$ versus $B_{\mathrm{W}}-R$ color-color diagram. Since we do not require the object to be well detected $(10 \sigma$ detection) in all three bands, as we did in Figure 6 , the stellar locus and galaxy distribution have greater scatter than those in Figure 6, which contributes to the contamination of the LBG sample. Using the selection criteria discussed in Section 4.2 , a total of 14,485 photometrically selected LBGs (cross symbols in Figure 8) are selected down to $R=25.0$ in the $8.8 \mathrm{deg}^{2}$ area, leading to an LBG surface density $\Sigma=0.47 \pm$ 0.03 galaxies $\operatorname{arcmin}^{-2}$. This value is smaller than the result, $\Sigma \sim 1.8$ galaxies $\operatorname{arcmin}^{-2}$, in Steidel et al. (2003), which is 0.5 magnitude deeper than our survey. Figure 9 shows that the surface number density of the $z \sim 3$ LBGs in this work is systematically lower than that in Reddy et al. (2008). The low number density is mainly due to the narrower redshift selection function in our sample compared to that in Reddy et al. (2008). Our shallower survey also increases the photometric errors and decreases the detection rate for a given magnitude, which reduces the completeness.

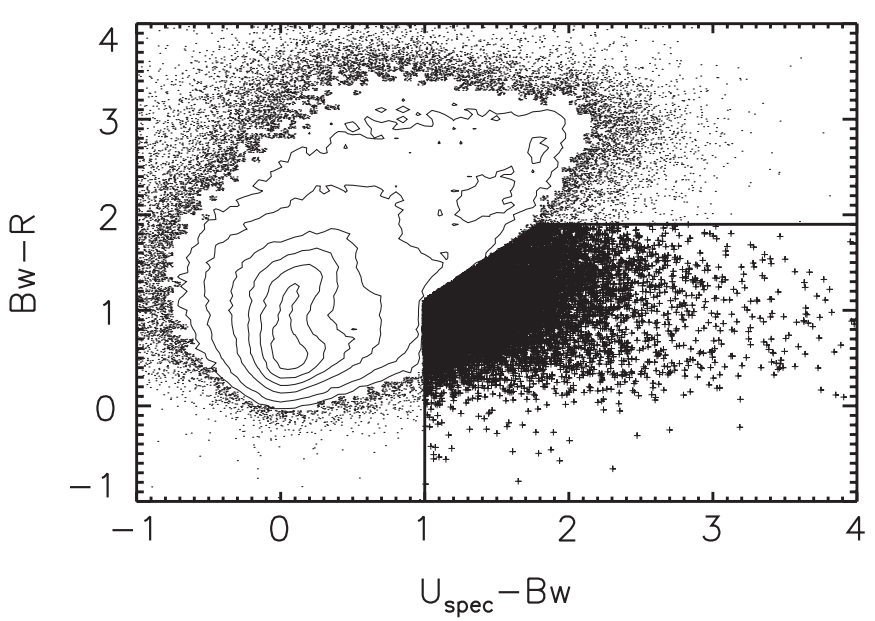

Figure 8. $B_{\mathrm{W}}-R$ vs. $U_{\text {spec }}-B_{\mathrm{W}}$ color-color diagram. The photometrically selected LBGs at $z \sim 3$ are selected in the region bounded by the solid line. A total of 14,485 photometrically selected LBGs are selected as U-dropouts. The crosses represent the selected LBGs. The reason for the sharp edge at $B_{\mathrm{W}}-R=1.9$ and $U_{\text {spec }}-B_{\mathrm{W}}=1.0$ is that we do not plot the $U_{\text {spec }}$-band undetected sources without falling in the selection criterion region.

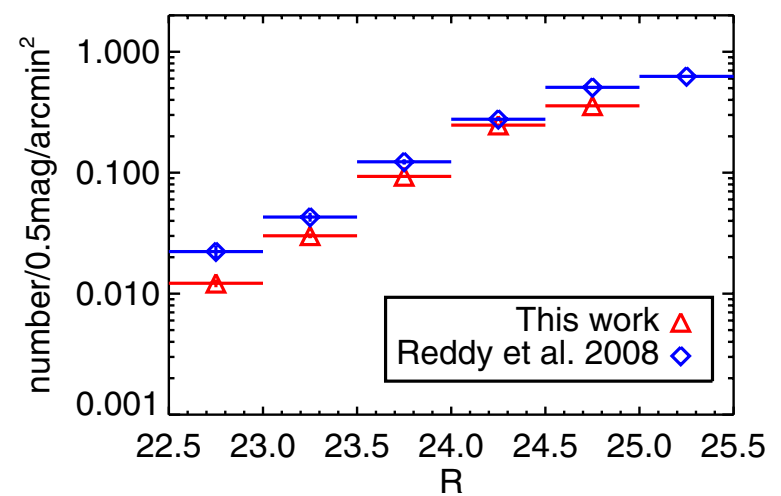

Figure 9. Surface density of $z \sim 3$ LBGs as a function of $R$-band magnitude in this work (red triangles) and in Reddy et al. (2008; blue diamonds).

(A color version of this figure is available in the online journal.)

\section{UV LUMINOSITY FUNCTION}

In this section, we will measure the rest-frame UV LF of LBGs based on their $R$-band magnitudes.

\subsection{Results}

The $R$-band filter $\left(\lambda_{\text {eff }}=6407 \AA\right)$ traces the rest-frame UV $(\sim 1700 \AA)$ for this LBG sample with a mean redshift of $z \sim 2.9$. The following formula is used to convert the apparent $R$-band AB magnitude $\left(m_{R}\right)$ to the absolute magnitude at rest-frame $1700 \AA\left(M_{1700 \AA}\right)$,

$$
\begin{aligned}
M_{1700 \AA}= & m_{R}-5 \log _{10}\left(d_{\mathrm{L}} / 10 \mathrm{pc}\right)+2.5 \log _{10}(1+z) \\
& +\left(m_{1700}-m_{\lambda_{\mathrm{obs}}} /(1+z)\right),
\end{aligned}
$$

where $d_{\mathrm{L}}$ is the luminosity distance in pc and $z$ is the redshift. The second and third terms of the right side are the distance modulus, and the fourth term is the $K$-correction between restframe $1700 \AA$ and the $R$-band, which is about 0 (Sawicki \& Thompson 2006a). From the simulation in Section 4.3, the mean redshift of the LBG sample is 2.9, which corresponds to a distance modulus of 45.46 .

The rest-frame $1700 \AA \operatorname{LF}\left(\Phi\left(M_{1700 \AA}\right)\right)$ and its statistical uncertainty in a given magnitude bin can be computed based on 
Schmidt (1968):

$$
\Phi\left(M_{1700 \AA}\right)=\frac{1}{\Delta m} \frac{N(1-f)}{V_{\text {eff }}},
$$

and

$$
\Delta \Phi\left(M_{1700 \AA}\right)=\frac{1}{\Delta m} \frac{\sqrt{N(1-f)}}{V_{\text {eff }}},
$$

where $\Delta m$ is the magnitude bin size, which is 0.5 in this paper, $N$ is the number of U-dropout LBG candidates falling into the magnitude bin, and $f$ is the fraction of contamination for the magnitude bin. In this paper, we do not have any spectral observations of these candidates. Therefore, we adopt the contamination fraction, $f$, from Table 3 in Reddy et al. (2008). The value of $f$ is about 0.7 at the bright end and less than 0.01 at the faint end. We will discuss how the contamination rate affects our LF measurements later in Section 5.3. $V_{\text {eff }}$ is the effective comoving volume in units of $\mathrm{Mpc}^{3}$.

In a flat universe, the comoving volume per solid angle per redshift can be calculated as

$$
\frac{d V}{d \Omega d z}=\frac{\operatorname{cr}(z)^{2}}{H(z)},
$$

where $r(z)=\int c d z / H(z)$. The effective comoving volume $\left(\mathrm{V}_{\text {eff }}\right)$ can be calculated from the comoving volume,

$$
V_{\mathrm{eff}}=\int_{\Delta z} \int_{\Delta m} d z d m P(m, z) \frac{V(z)}{d z d m},
$$

where $P(m, z)$ is the completeness of the sample as a function of redshift $(z)$ and $R$-band magnitude $(m)$, which has been determined in Section 4.3. The effective volume for a given magnitude is computed by integrating Equation (9) using the results of the selection function $P(m, z)$. Then the LF is calculated for each magnitude bin.

The red triangles in Figure 10 represent the UV LF measurement result of our LBG sample. The UV LF is fitted by the Schechter function:

$$
\begin{aligned}
\Phi\left(M_{1700 \AA}\right) d M(1700 \AA)= & \frac{2}{5} \Phi^{\star} \ln (10)\left[10^{\frac{2}{5}\left(M^{\star}-M\right)}\right]^{\alpha+1} \\
& \times \exp \left[-10^{\frac{2}{5}\left(M^{\star}-M\right)}\right] d M,
\end{aligned}
$$

and the best-fit parameters for the Schechter function are $\Phi^{\star}=(1.06 \pm 0.33) \times 10^{-3} \mathrm{Mpc}^{-3}, M^{\star}=-21.11 \pm 0.08$, and $\alpha=-1.94 \pm 0.10$. For the LF fitting, we do not use the LF in the magnitude bins brighter than $M_{1700 \AA}=-23$, as they are significantly overestimated due to contamination by quasars. The survey depth of the Boötes field is only about 0.5 magnitude fainter than $M^{\star}$, thus these data cannot be used to constrain the faint-end slope of the LF very well. Therefore, we combined our LF measurement with the LF at $M(1700 \AA)>-20.5$ from Reddy \& Steidel (2009) to fit the Schechter function (10). We find that the best-fit parameters are $\Phi^{\star}=(1.12 \pm 0.17) \times 10^{-3} \mathrm{Mpc}^{-3}, M^{\star}=-21.08 \pm 0.05$, and $\alpha=-1.90 \pm 0.05$. Combining the LF measurements from different data sets could bring significant systematic errors into the LF fitting due to the quite different filter sets and selection criteria. In Figure 10, we compared the LF derived in this work with those in Reddy \& Steidel (2009), Poli et al. (2001), Arnouts et al. (2005), and van der Burg et al. (2010) in the magnitude range $-23<M(1700 \AA)<-20.5$. They are consistent with each other within $1 \sigma$.

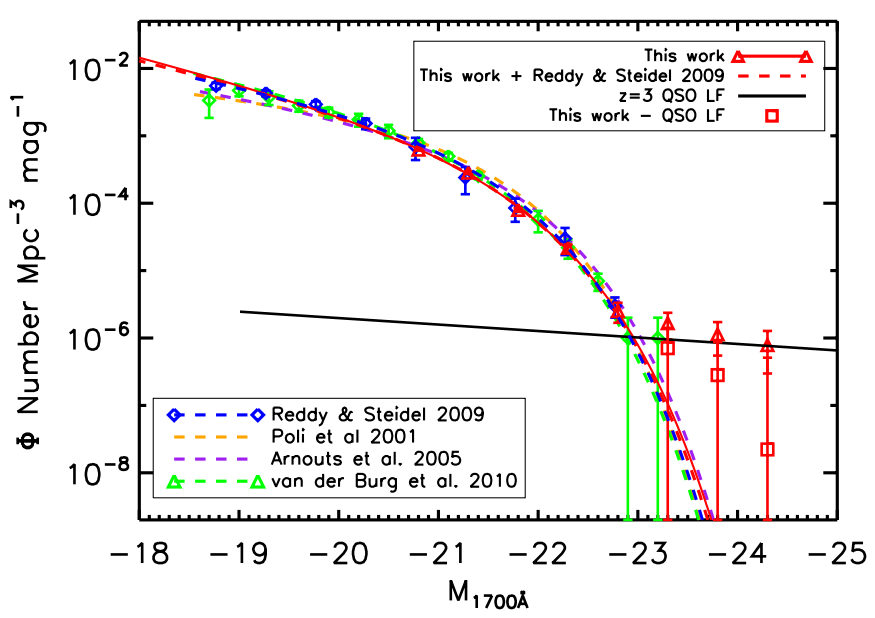

Figure 10. Rest-frame UV (1700 ̊) luminosity function (LF) of the $z \sim 3$ LBGs in Boötes field (red triangles) and the best-fit Schechter function (red dashed and solid curves). For comparison, we also plot the LFs of $z \sim 3$ LBGs from Reddy \& Steidel (2009; the blue diamonds and the dashed curve), Poli et al. (2001; orange dashed curve), Arnouts et al. (2005; purple dashed curve), and van der Burg et al. (2010; green triangles and dashed curve). All these works are consistent with each other at the luminosity range, $-22.5<M_{1700 \AA}<-20.5$. The bright end of the LF in this work shows excess compared with the Schechter function. The excess can be explained by the $z \sim 3$ quasar LF (black solid line; Hunt et al. 2004). The red squares represent the residual values that quasar LF is subtracted from the LBG LF measurements.

(A color version of this figure is available in the online journal.)

\subsection{UV Luminosity Density}

The UV luminosity density from integrating the Schechter function for a faint luminosity limit is given by

$$
\rho_{L_{\mathrm{UV}}}=\left[\Gamma(\alpha+2)-\gamma\left(\alpha+2, L_{\mathrm{lim}} / L^{\star}\right)\right] \Phi^{\star} L^{\star},
$$

where $\Gamma(x)=\int_{0}^{+\infty} e^{-t} t^{x-1} d t$, and $\gamma(x, l)=\int_{0}^{l} e^{-t} t^{x-1} d t$. To compare with previous results (e.g., Sawicki \& Thompson $2006 \mathrm{~b}$ and references therein), the faint luminosity limit is set as $0.1 L^{\star}$. The luminosity density at $1700 \AA$ can be computed from

$$
L_{1700 \AA}=\frac{4 \pi d_{L}^{2}}{1+z} 10^{-\frac{2}{5}\left(48.6+m_{R}\right)} .
$$

The characteristic luminosity, $L_{1700 \AA}^{\star}=1.2 \times 10^{29} \mathrm{erg} \mathrm{s}^{-1} \mathrm{~Hz}^{-1}$ based on our best Schechter function fit. Both the LF measured by this work and the LF measured by combining this work and the faint-end data points from Reddy \& Steidel (2009) are used to compute the UV luminosity density. The total UV luminosity density derived from these two measurements are consistent with each other, which are $2.19 \pm 0.08 \times 10^{26} \mathrm{erg} \mathrm{s}^{-1} \mathrm{~Hz}^{-1} \mathrm{Mpc}^{-3}$ and $2.18 \pm 0.05 \times$ $10^{26} \mathrm{erg} \mathrm{s}^{-1} \mathrm{~Hz}^{-1} \mathrm{Mpc}^{-3}$, respectively. In Table 2, we summarize the results of LF measurements and the total UV luminosity density from this work, Reddy \& Steidel (2009), Shim et al. (2007), and Sawicki \& Thompson (2006a, 2006b). We find that our UV luminosity density results agree with that from Reddy \& Steidel (2009) within $1 \sigma$ uncertainty, but are significantly larger than that from Sawicki \& Thompson (2006a, 2006b; by about $6 \sigma$ ) and from Shim et al. (2007). This discrepancy is mainly due to the different faint-end slopes estimated. Compared to the results from Sawicki \& Thompson (2006a, 2006b) and Shim et al. (2007), this work and Reddy \& Steidel (2009) suggest a much steeper faint-end slope of the UV LF (Table 2), and that the faint LBGs make a significant contribution to the UV luminosity density. 
Table 2

Schechter Parameters of UV LFs and Luminosity Densities

\begin{tabular}{lccccc}
\hline \hline Redshift & $\alpha$ & $M^{\star}(1700 \AA)$ & $\begin{array}{c}\Phi^{\star}\left(\times 10^{-3}\right) \\
\left(\mathrm{Mpc}^{-3}\right)\end{array}$ & $\begin{array}{c}\rho_{L_{\mathrm{UV}}}\left(\times 10^{26}\right) \\
\left(\mathrm{erg} \mathrm{s}^{-1} \mathrm{~Hz}^{-1} \mathrm{Mpc}^{-3}\right)\end{array}$ \\
\hline $2.7<z<3.3$ & $-1.94 \pm 0.10$ & $-21.11 \pm 0.08$ & $1.06 \pm 0.33$ & $2.19 \pm 0.08$ & Ref \\
$2.7<z<3.3$ & $-1.90 \pm 0.05$ & $-21.08 \pm 0.05$ & $1.12 \pm 0.17$ & $2.18 \pm 0.05$ & This work + Reddy \& Steidel (2009) \\
$2.7<z<3.4$ & $-1.73 \pm 0.13$ & $-20.97 \pm 0.14$ & $1.71 \pm 0.53$ & $2.55 \pm 0.25$ & Reddy \& Steidel (2009) \\
$3.0<z<3.4$ & -0.83 & -20.69 & 1.54 & 1.15 & Shim et al. (2007) \\
$2.7<z<3.3$ & $-1.43_{-0.09}^{+0.17}$ & $-20.90_{-0.14}^{+0.22}$ & $1.70_{-0.25}^{+0.59}$ & $1.81 \pm 0.04$ & Sawicki \& Thompson (2006a, 2006b) \\
\hline
\end{tabular}

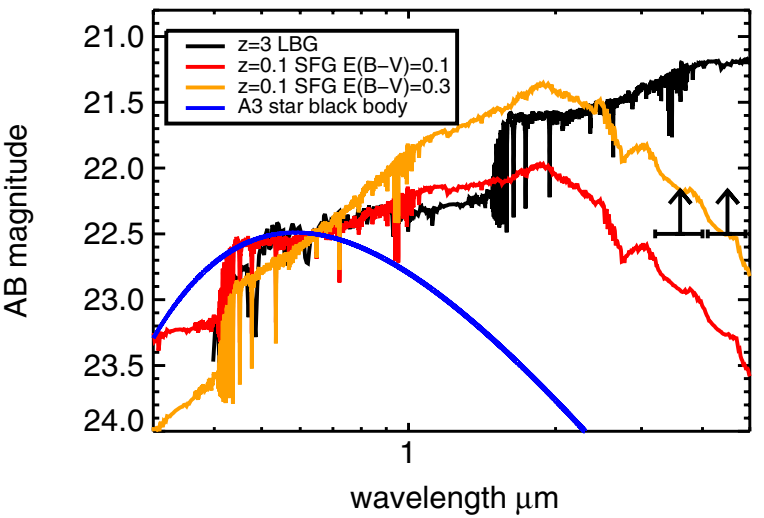

Figure 11. Spectra of $z \sim 3$ LBGs and low-redshift interlopers: $z=0.1$ starforming galaxies (SFGs) with $E(B-V)=0.1$ and $E(B-V)=0.3$ and an A-type star. All the spectra are scaled to the $R=22.5$. The two upward arrows represent the $5 \sigma$ flux limits of the [3.6] and [4.5] band in Boötes field.

(A color version of this figure is available in the online journal.)

\subsection{Systematic Uncertainties of LF Measurement}

The following effects are considered for their impact on the LF measurements, especially in term of the possibility of introducing systematic errors.

1. Contamination fraction:for the LF measurement, we use the fraction of low-redshift interlopers from Reddy et al. (2008), in which the spectroscopically confirmed sample is used to constrain the contamination rate in each magnitude bin. At the faint end, the fraction approaches zero, while it is about $70 \%$ at the bright end. The reasons why the contamination rate is low at the faint end are (1) Galactic type A stars are not as faint as $R=25$; and (2) the LF of low-redshift galaxies $(z \sim 0.1)$ becomes flat at the faint end and the survey volume at $z \sim 3$ is about two orders of magnitude higher than that at $z \sim 0.1$.

We use Spitzer IRAC photometry to estimate the brightend contamination rate of low-redshift interlopers. The majority of the interlopers are A-type stars and star-forming galaxies at $z \sim 0.1$. Figure 11 shows the observed-frame model SED of $z \sim 3$ LBGs and those two types of interlopers in the wavelength range from $3000 \AA$ to $5 \mu \mathrm{m}$. The spectra of star-forming galaxies are generated using the same procedure as described in Section 4.2 and then shifted to $z=0.1$ and $z=3$. Figure 11 suggests that the interlopers, both A-type stars and star-forming galaxies at $z=0.1$, are expected to have bluer $R-[3.6]$ and [3.6]-[4.5] colors than $z \sim 3$ LBGs. We use the $R-$ [3.6] and [3.6] $-[4.5]$ colors to estimate the contamination rate of galaxy candidates in the $R$-band magnitude range between 22.0 and 22.5. The magnitude limit in the [3.6] band is about $22.5 \mathrm{AB}(5 \sigma)$. As shown in Figure 11, the bright $z \sim 3$ LBGs should have firm detections in both the
[3.6] and [4.5] bands (e.g., Bian et al. 2012), however, neither A-type stars nor $z=0.1$ star-forming galaxies with $E(B-V)=0.1$ can be detected in both the [3.6] and [4.5] bands. Star-forming galaxies at $z=0.1$ with $E(B-V)=0.3$ can be detected in the [3.6] band and marginally in the [4.5] band, but they have much bluer $R$ [3.6] and [3.6]-[4.5] colors than those in LBGs. There are about 360 LBG candidates in the $R$-band magnitude range between 22.0 and 22.5; among them, about 110 candidates have both [3.6] and [4.5] detections and [3.6]-[4.5] $>0.0$. The latter color cut will exclude most of the galaxies/AGNs lower than $z \sim 1.5$ (e.g., Donley et al. 2008). Our followup spectroscopic observations have shown that this color cut is very robust in rejecting contamination from lowredshift interlopers (F. Bian et al. 2013, in preparation). This result indicates that the contamination rate of low-redshift interlopers in the bright LBG candidates is indeed about $70 \%$. We cannot distinguish AGNs/quasars from LBGs at $z \sim 3$ using [5.8] - [8.0] color (Lacy et al. 2004; Stern et al. 2005; Donley et al. 2008) due to the shallow [5.8] and [8.0] imaging data.

2. Redshift distribution: when we calculate the luminosity of LBGs, all LBGs are assumed to be at the same redshift $(z=2.9)$ rather than in a redshift distribution. The FWHM of the redshift distribution (Figure 7) is about 0.6 $(2.7<z<3.3)$, which scatters the LBGs from a given absolute magnitude bin into neighboring magnitude bins. There are more LBGs in the fainter absolute magnitude bin, introducing a bias, especially at the bright end. To estimate the influence of this effect, we conduct a Monte Carlo (MC) simulation. We focus on the 22.0-22.5, 22.5-23.0, and 23.0-23.5 mag bins. Each magnitude bin is divided into 10 sub-bins. The number of galaxies is generated for each sub-bin following the Schechter function (Equation (10)), and the scatter in apparent magnitude is mainly due to the redshift distribution in Figure 7. The final number of galaxies in the 22.5-23.0 mag bin is compared with the initial number of galaxies falling into this magnitude bin, and we find that the LF for the 22.5-23.0 mag bin can be overestimated by $\sim 18 \%$ due to this effect. For comparison, the statistical error for this magnitude bin is $7 \%$.

3. Galaxy spectral template model: the effective comoving volume calculation depends on the galaxy spectral template. The spectral template used in our simulation is a spectrum of an idealized galaxy with a 300 Myr old stellar population and a constant SFR, solar metallicity, and Salpeter IMF, and an $E(B-V)$ distribution from Reddy et al. (2008). All these parameters can affect the value of $\mathrm{V}_{\text {eff }}$. We perform an $\mathrm{MC}$ simulation to estimate the effective comoving volume with different ages (from $100 \mathrm{Myr}$ to $1 \mathrm{Gyr}$ ) and find that the effective comoving volumes change by less than $5 \%$ due to the age, which is consistent with the results 
in Sawicki \& Thompson (2006a). Another factor that introduces uncertainty is the distribution of $E(B-V)$. We allow the fraction of the LBGs in each $E(B-V)$ bin to vary by $20 \%$ and perform an MC simulation, and find that the uncertainty in the LF caused by the $E(B-V)$ variation is less than $10 \%$. The spectral template does not include the Lyman $\alpha$ emission/absorption, which could also influence our results. In the redshift range to which the selection criteria are sensitive, the Ly $\alpha$ line falls into the $B_{\mathrm{W}}$-band filter. For an LBG with observed-frame Ly $\alpha$ equivalent width of $50 \AA$, the real intrinsic $U_{\text {spec }}-B_{\mathrm{W}} /$ $B_{\mathrm{W}}-R$ color will be 0.05 magnitude redder/bluer than that in our simulation, which will make galaxies at lower redshift fall into the selection criterion, but the difference is much smaller than the typical uncertainty of a $U_{\text {spec }}$-band magnitude $(\approx 0.5)$ or a $B_{\mathrm{W}}$-band magnitude $(\approx 0.1)$. This effect will influence the effective volume estimation by less than $3 \%$. Therefore, neither the galaxy age in the template spectra nor the Ly $\alpha$ emission/absorption line makes a large impact on the selection function.

4. Cosmic variance: cosmic variance is another possible source of systematic uncertainty for the LF measurement due to the limited survey volume and the fluctuations of the dark matter density on large scales. Using the cosmic variance calculator $^{11}$ (Trenti \& Stiavelli 2008), we find that the cosmic variance for the Boötes field is about $4 \%$ for a minimum halo mass of $8 \times 10^{11} M_{\odot} h^{-1}$. For comparison, the cosmic variance in a one $\mathrm{deg}^{2}$ area is $9 \%$ for a minimum halo mass of $8 \times 10^{11} M_{\odot} h^{-1}$. Cosmic variance dominates the uncertainty of the LF at the bright end compared to Poisson errors.

We conclude that the greatest degree of uncertainty in the LF measurement comes from the contamination fraction; all other factors combined will contribute $\lesssim 30 \%$ uncertainty to the LF measurement.

\subsection{Discussion}

The large survey field also allows us to select a sizable sample of luminous LBGs with $-23>M_{1700 \AA}>-25$ $\left(L>6 L^{\star}\right)$, probing the UV LF in this range for the first time. Our measurement (the brightest three points) shows an excess power compared to the Schechter function fit. van der Burg et al. (2010) found a similar excess power in the luminosity range $-23>M_{1700 \AA}>-23.5$ (green triangles in Figure 10), and they suggested that it is due to gravitational lensing. In Figure 10, we show the $z \sim 3$ quasar LF (Hunt et al. 2004) and find that the bright-end LBG LF follows the quasar LF well, which suggests that the majority of the excess power can be explained by the LF of $z \sim 3$ quasars. We subtract the quasar LF value from these three points to statistically remove the quasar contribution. The three red squares present the residual values, which are still higher than the best-fit Schechter function. It is worth noting that the uncertainty of the quasar LF measurements from Hunt et al. (2004) is large due to the small size of the faint quasar sample. Therefore, the excess power of the LBG LF can be within the uncertainty of the quasar LF measurements. If the excess is real, it can be caused by gravitational lensing, which boosts the fainter LBGs to the bright end (e.g., Jain \& Lima 2011). It is also possible that the LF of LBGs actually shows excess power at the bright end. The similar excess power at the

\footnotetext{
11 http://casa.colorado.edu/ trenti/CosmicVariance.html
}

bright end ( $L>2 L^{\star}$ ) of the UV LF has been found in the $z \sim 7-8$ LBG sample (e.g., Yan et al. 2011, 2012). The brightend cutoff of the UV LF is regulated by feedback processes and dust obscuration (e.g., Lacey et al. 2011). If the excess power is real, that would suggest that those physical mechanisms are probably not efficient in these most UV luminous LBGs. To have an accurate measurement of the bright-end LF, followup spectroscopic observations for the bright LBGs are required (F. Bian et al. 2013, in preparation).

\section{NEAR-IR LUMINOSITY FUNCTION}

The Spitzer Deep-Field Survey (Ashby et al. 2009) covers the whole $9 \mathrm{deg}^{2}$ NDWFS Boötes field with all four IRAC bands at wavelengths of $3.6 \mu \mathrm{m}, 4.5 \mu \mathrm{m}, 5.8 \mu \mathrm{m}$, and $8.0 \mu \mathrm{m}$. There are four epochs in the survey with a total exposure time of $12 \times 30 \mathrm{~s}$. In this paper, we use the IRAC2 (4.5 $\mu \mathrm{m},[4.5])$ band to probe the rest-frame near-IR emission of LBGs at $z \sim 3$. The advantages of the [4.5] band are: (1) the depth of the [4.5] band is comparable to the [3.6]-band depth; (2) the [4.5] band is less influenced by the AGN power-law component than the [5.8] and [8.0] bands; and (3) the rest-frame wavelength of [4.5] at $z \sim 3$ is at $1.1 \mu \mathrm{m}(J$-band at $z \sim 0)$, which probes the evolved stellar population and is not affected by strong emission lines that bias the observed-frame IR continuum measurements. As discussed in Section 5.3, the [4.5] detection rate is high $(\sim 100 \%)$ in the bright LBGs $(R<23.0)$. The detection rate decreases quickly with increasing magnitude, and the [4.5]-band detection rate for the faintest magnitude bin $(24.5<R<25.0)$ is about $50 \%$. The rest-frame near-IR LF is derived based on the UV LF and the relation between the $R$-band magnitude and the $R-$ [4.5] color in the LBGs at $z \sim 3$. We follow the method developed by Shapley et al. (2001), who estimated the $K$-band (rest-frame $V$-band) LF of LBGs at $z \sim 3$ based on their UV LF and UV-optical colors.

\subsection{The Rest-frame UV/Near-IR Color Relation of LBGs}

We carry out photometry on the [4.5]-band images using SExtractor with the same parameters as in Table 2 of Ashby et al. (2009), except that we use a lower detection threshold $($ DETECT_THRESH $=1.0)$ to detect fainter sources. We use the $4^{\prime \prime}$ aperture magnitude as the output magnitude. We first apply the aperture correction to the magnitude and then covert the Vega magnitude to AB magnitude. The $2.5 \sigma$ limiting magnitude is $23.4 \mathrm{AB}$. Finally, the positions of sources are matched with the LBG positions.

The distribution of $R$-band magnitude versus $R-$ [4.5] color is shown in Figure 12. The solid line represents the magnitude limit of the [4.5] band. As there are only upper limits of $R-$ [4.5] color for a large fraction of LBGs, we used one of the survival analysis methods, the schmidttbin method based on maximum likelihood estimation (Schmitt 1985), in the Astronomy Survival Analysis (ASURV; Isobe et al. 1986) in the IRAF package, stsdas.analysis.statistics, to take into account the upper limits of $R-$ [4.5] color. We calculated the linear regression between $R$ and $R-[4.5]$ color. and found that the slope, $\Delta(R-[4.5]) / \Delta R$, is 0.22 . The probability of correlation between $R$ and $R-$ [4.5] color is greater than $99.98 \%$. We also use other methods in the ASURV package, e.g., emmethod and buckleyjames method, and obtain similar results.

\subsection{Results}

We estimate the near-IR LF using simulated galaxy colors based on the UV LF measurements and the rest-frame 


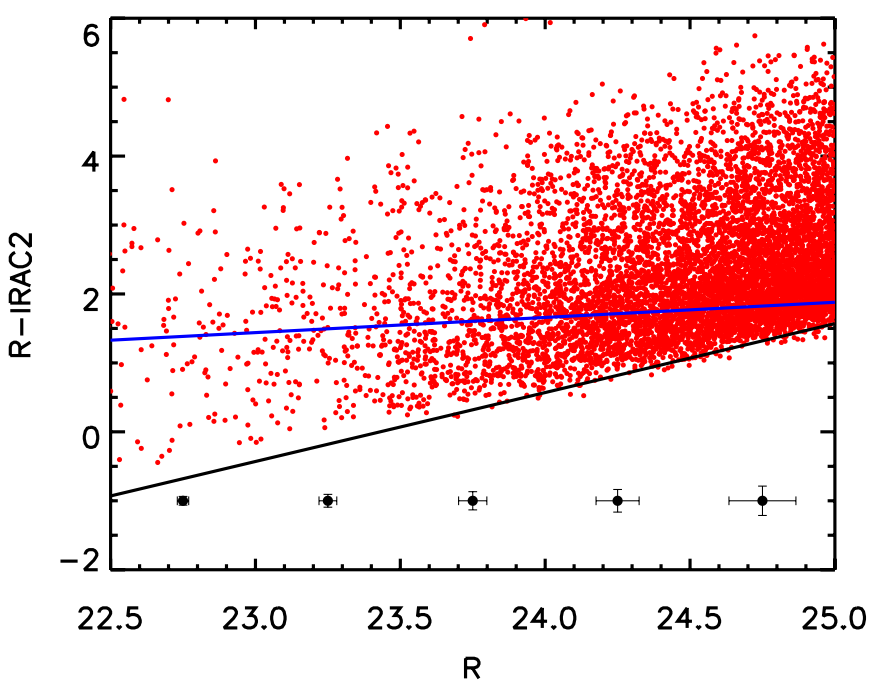

Figure 12. Relation between $R$ magnitude and $R-$ [4.5] color (red filled points) The black solid line shows the typical magnitude limits of the [4.5] band, and the blue solid line represents the best-fitted linear regression line with survival analysis method. The black filled points with error bar represent typical errors of $R$-band magnitude and $R-$ [4.5] color in the individual magnitude bins.

(A color version of this figure is available in the online journal.)

$\mathrm{UV} /$ near-IR color relation, following Shapley et al. (2001): (1) we generate 100,000 galaxies with $R$-band magnitudes in the range from 22.5 to 27.0 . The distribution of the $R$-band magnitudes follows the distribution of the UV LF in Section 5.1; (2) we derive the distribution of $R-[4.5]$ in the $23.4<R<23.5$ magnitude bin. In this magnitude bin, the [4.5]-band detection rate is about $75 \%$, and the quasar/AGN contamination rate is low. We assume the $R-$ [4.5] distribution is Gaussian. At $R \approx 23.5$, the $R-[4.5]$ color distribution is truncated at $R-[4.5] \approx 0$ due to the detection limit of the [4.5] band. Therefore, we only use galaxies with $R-$ [4.5] color values in the range between 0 and 4 to fit the Gaussian function; (3) we generate 100,000 $R-$ [4.5] colors that follow the Gaussian distribution function derived in step (2). For each $R-[4.5]$ value, an offset $(\Delta(R-[4.5]))$ is applied based on its $R$-band magnitude, $\Delta(R-[4.5])=0.22(R-23.45)$, to get the final relation between $R$-band magnitude and [4.5] magnitude for each galaxy. Using this method, the [4.5] magnitudes for a sample of 100,000 galaxies are generated based on their $R$-band magnitude and the relation between $R$ magnitude and $R-$ [4.5] color, and the [4.5]-band LF is derived; (4) we use a MC simulation to estimate the uncertainty of the near-IR LF. 10,000 simulated UV LFs are generated based on the uncertainty of the UV LF measurements in Section 5.1. The same procedure as above is used to transfer the $R$-band magnitude to the [4.5] magnitude in $z \sim 3$ LBGs. When we use the $R$-band magnitudes to calculate the [4.5] magnitudes, the uncertainties, including the $R$-band and [4.5]-band photometric errors, the uncertainty of the slope between $R$ and $R-$ [4.5] color, and the uncertainty of the Gaussian distribution of the $R-$ [4.5] color for the given $R$ magnitude range, are also considered. Finally, a total of 100,000 simulated near-IR LFs are derived. The standard deviations of the 10,000 near-IR LFs in each magnitude bin are considered as the uncertainties of the near-IR LF; and (5) we fit the [4.5]-band (rest-frame 1.1 $\mu \mathrm{m}$ ) LF with a Schechter function (Figure 13) and find that the best-fit Schechter function parameters for the rest-frame $1.1 \mu \mathrm{m} \mathrm{LF}$ of $z \sim 3 \mathrm{LBGs}$ are $\phi_{J}^{\star}=(3.1 \pm 1.9) \times 10^{-4} \mathrm{Mpc}^{-3}, M_{J}^{\star}=-24.00 \pm 0.30$, and $\alpha_{J}=-2.00 \pm 0.17$.

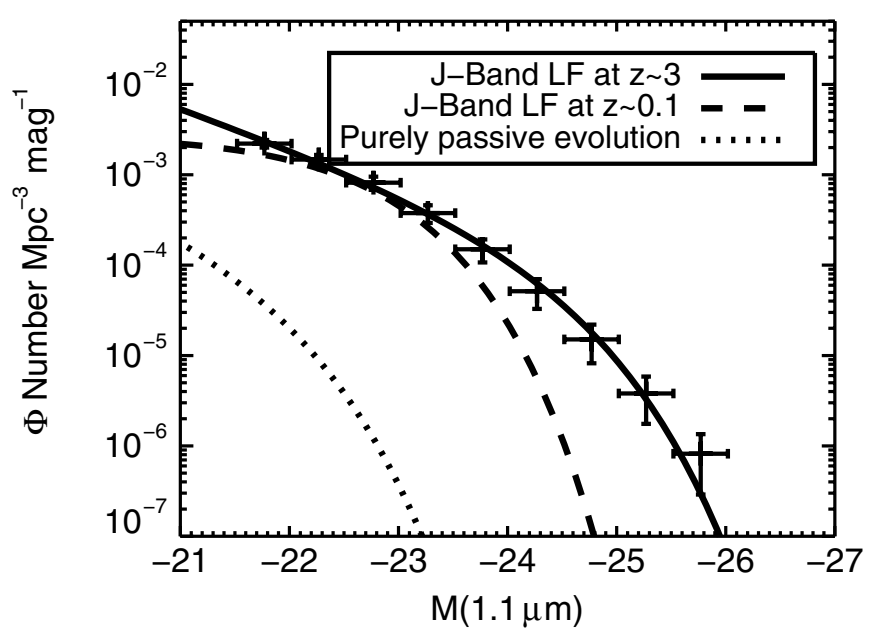

Figure 13. $z \sim 3$ LBG LF of [4.5] band (rest-frame $J$-band). The solid line represents the best-fit Schechter function for the [4.5]-band (rest-frame $1.1 \mu \mathrm{m}$ ) $\mathrm{LF}$, and the dashed line represents the $J$-band LF for the galaxy in the local universe. The dotted line is the predicted $J$-band LF at $z \sim 0.1$, if we assume a purely passive evolution in these galaxies from $z \sim 3$.

\subsection{Discussion}

Figure 13 compares the rest-frame $1.1 \mu \mathrm{m}$ LF of $z \sim 3$ LBGs to the $J$-band LF of nearby galaxies at $z \sim 0.1$ (Cole et al. 2001). The best-fit Schechter function parameters for nearby galaxies are $\phi_{J}^{\star}=(3.57 \pm 0.36) \times 10^{-3} \mathrm{Mpc}^{-3}, M_{J}^{\star}=-23.13 \pm 0.02$, and $\alpha_{J}=-0.93 \pm 0.04$. The rest-frame $J$-band LFs show significant evolution from $z=3$ to $z \sim 0.1$. Compared to the local LF, the rest-frame $J$-band LF at $z \sim 3$ has smaller $\phi_{J}^{\star}$ by an order of magnitude, a steeper faint-end slope $(\alpha)$, and brighter characteristic luminosity $\left(M_{J}^{\star}\right)$ by $\approx 1.0$ magnitude.

These trends are also found in the rest-frame optical ( $V$-band) LF between $z \sim 3$ and $z \sim 0$ (Shapley et al. 2001). The steep faint-end slope of the rest-frame near-IR LF is mainly due to the steep faint-end slope of the rest-frame UV LF and the positive correlation between $R$ magnitude and $R-$ [4.5] color. A similarly steep faint-end slope $(\alpha=-1.85 \pm 0.15)$ is also found in the optical ( $V$-band) LF of LBGs at $z \sim 3$ (Shapley et al. 2001), which is consistent within the errors with our near-IR faint-end slope, and the optical LF in Shapley et al. (2001) is derived based on a UV faint-end slope of $\alpha \sim-1.57$. If the authors adopt a much steeper UV faint-end slope, such as that in Reddy \& Steidel (2009) and this work, the faint-end slope of the optical LF will get even steeper. One of the caveats for the rest-frame near-IR LF derived in this work is that the IRAC depth of the LBG sample is shallow. We obtain the $R-$ [4.5] color distribution based on a bright magnitude bin and assume that this distribution does not change in the fainter magnitude bins, therefore, it is unclear whether the $R-$ [4.5] color distribution and the positive slope between $R$ magnitude and $R-$ [4.5] color will still hold for the faint LBGs.

To derive the rest-frame $J$-band luminosity density of LBGs at $z \sim 3$, we integrate the Schechter function to a faint luminosity limit, 0.1 $L^{\star}$, which is about the magnitude limit of the [4.5]band images and find $\log _{10} \rho_{J}\left(\mathrm{erg} \mathrm{s}^{-1} \mathrm{~Hz}^{-1}\right)=27.04_{-0.11}^{+0.09}$.

We consider a simple purely passive evolution model for the near-IR LF. Using the BC03 model, we generate SEDs of galaxies with a constant star formation history of $300 \mathrm{Myr}$, which is about the typical age of LBGs at $z \sim 3$ (Shapley et al. 2001), then we shut down the star formation in the galaxies and make these galaxies passively evolve for the following 


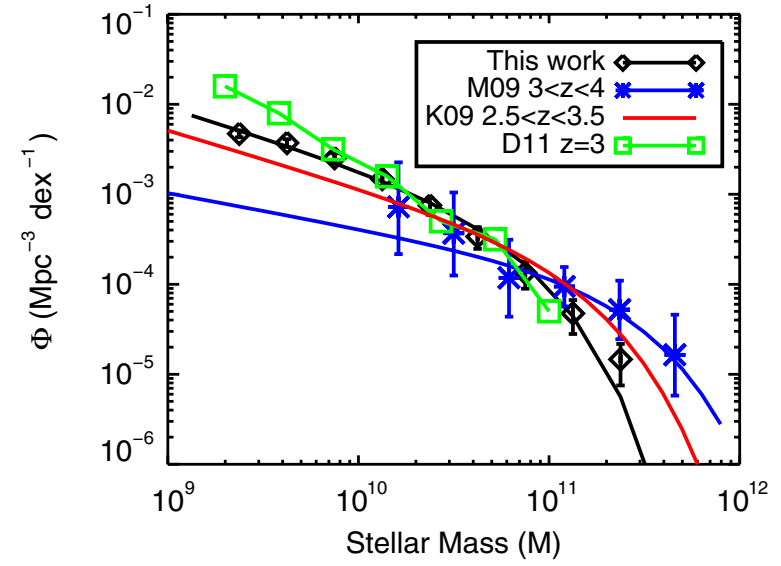

Figure 14. Stellar mass functions of $z \sim 3$ LBGs from this work (black solid line), and $K$-selected galaxies (Kajisawa et al. 2009; Marchesini et al. 2009, K09, red curve, M09, blue curve). The green curve represents the $z \sim 3$ stellar mass function derived from cosmological simulations with momentum-conserved wind feedback model (Davé et al. 2011, D11).

(A color version of this figure is available in the online journal.)

$11.0 \mathrm{Gyr}$, from $z \sim 3$ to $z \sim 0.1$. We find that the absolute $J$-band magnitude (after reddening with $E(B-V)=0.15$ ) at $z \sim 3$ right after shutting down star formation is about $2.7 \mathrm{mag}$ brighter than that at $z \sim 0$. In Figure 13, the dotted curve represents the predicted local near-IR LF based on this purely passive evolution model.

\subsection{Stellar Mass Function}

Since the near-IR is a good tracer of the old and evolved stellar population, the rest-frame near-IR LF can be used to derive the SMF of LBGs at $z \sim 3$. We adopt the relation between the rest-frame $1.1 \mu \mathrm{m}$ absolute magnitude and the stellar mass in Reddy et al. (2012):

$$
\log \left(M_{\text {stellar }} / M_{\odot}\right)=-(0.56 \pm 0.09) M_{1.1}-(2.42 \pm 1.94) .
$$

Using this relation, the faint-end slope of the SMF will be flatter compared to that of the rest-frame $J$-band LF. In Figure 14, we show the SMF and best-fit Schechter function of LBGs at $z \sim 3$. The best-fit parameters are $\phi^{\star}=(2.8 \pm 1.1) \times 10^{-4} \mathrm{Mpc}^{-3}$, $\log _{10} M_{\text {stellar }}^{\star}\left(M_{\odot}\right)=10.78 \pm 0.11$, and $\alpha=-1.65 \pm 0.09$. The large survey area allows us to reduce the cosmic variance, which contributes significant uncertainty to the previous SMF measurements (e.g., Marchesini et al. 2009). In Figure 14, we also show the SMFs derived from $z \sim 3 K$-selected galaxy samples in deep field surveys (Marchesini et al. 2009; Kajisawa et al. 2009) and the galaxy SMF at $z \sim 3$ predicted by cosmological hydrodynamic simulations (Davé et al. 2011). For comparison, we scale the galaxy stellar mass derived based on different IMFs to the mass based on a Kroupa IMF (Kroupa 2001). The $K$-selected galaxy sample in Marchesini et al. (2009) is selected from about $400 \operatorname{arcmin}^{2}$, which is about four times larger than that in Kajisawa et al. (2009); on the other hand, the survey depth in Kajisawa et al. (2009) is about 1-2 mag deeper than that in Marchesini et al. (2009). Therefore, Marchesini et al. (2009) put stronger constraints on the SMF at the high mass end, while Kajisawa et al. (2009) measure the low mass end of the SMF more accurately.

At the high mass end, $M_{\text {stellar }}^{\star}$ in the LBG sample is smaller than those in the $K$-selected galaxy samples (Marchesini et al. 2009; Kajisawa et al. 2009) at a $2 \sigma$ significance level, indicating a lower characteristic mass in the LBGs. For galaxies with stellar mass greater than $10^{11} M_{\odot}$, the density of LBGs is significantly smaller than that of mass selected galaxies, especially for the sample from Marchesini et al. (2009). We find that the ratio of stellar mass density between this work and Marchesini et al. (2009)/Kajisawa et al. (2009) is $0.26_{-0.15}^{+0.20} / 0.38_{-0.26}^{+0.62}$ in the stellar mass range $10^{11}-10^{12} M_{\odot}$, which suggests that LBGs are not the dominant galaxy population at the high mass end of the galaxy SMF at $z \sim 3$. LBGs are selected based on rest-frame UV colors; therefore, this method cannot select highly obscured galaxies or passively evolving early-type galaxies. van Dokkum et al. (2006) study a sample of massive $K$-selected galaxies $\left(M_{\text {stellar }}>10^{11} M_{\odot}\right)$ and find that the LBGs make up only $20 \%$ of the sample, and the rest of the sample are distant red galaxies (Franx et al. 2003).

At the low mass end, the SMF shows a somewhat steeper slope, although this difference is statistically insignificant. The value of the slope agrees with that in Marchesini et al. (2009; $\alpha=$ $\left.-1.39_{-0.55}^{+0.63}\right)$ and Kajisawa et al. $\left(2009 ; \alpha=-1.63_{-0.15}^{+0.14}\right)$ within $1 \sigma$ error. This suggests that the LBG population dominates the low mass end of the galaxy SMF at $z \sim 3$. This steep slope also suggests that the UV-selected star-forming galaxies make significant contributions to the total stellar mass density (e.g., Kajisawa et al. 2009; Reddy \& Steidel 2009). By integrating the LBG SMF, we find the stellar mass density of $z \sim 3$ LBGs with stellar mass between $10^{8}$ and $10^{10} M_{\odot}$ to be $51 \pm 4 \%$ of the total $z \sim 3$ LBG stellar mass density, which agrees with the result in Reddy \& Steidel (2009).

In the mass range between $10^{9.5}$ and $10^{11} M_{\odot}$, the SMF of LBGs is roughly consistent with that derived from cosmological hydrodynamic simulations (Davé et al. 2011). In this type of simulation, stellar mass is regulated by momentum-driven winds (Murray et al. 2005a) and recycled wind mode accretion (Oppenheimer et al. 2010). At the low mass end, the model overproduces the number of the galaxies, showing a steeper slope with $\alpha=-2.0$. On the other hand, the SMF at the massive end predicted by the model is consistent with that of LBGs, but is smaller than that in mass selected galaxies; this may reflect the finite simulation volume that under-predicts the massive galaxy population.

\section{THE EVOLUTION OF THE UV LF AND SMF}

We compare our $z \sim 3 \mathrm{UV}$ LF and SMF results with those from other high-redshift LBG $(z>4)$ studies (Bouwens et al. 2007; Lee et al. 2012) to study the evolution of the UV LF and SMF with cosmic time. Figure 15 shows how the best-fit Schechter function parameters, including dust-corrected $M_{\mathrm{UV}}^{\star} / M_{\text {stellar }}^{\star}, \phi^{\star}$, and $\alpha$ in UV LF and SMF, evolve with redshift. For the UV LF evolution, the black open triangle at $z \sim 3$ is from this work. The LF measurements for LBGs at $z \sim 4$, $z \sim 5$, and $z \sim 6$ are from Bouwens et al. (2007), and the data point at $z \sim 7$ is from Bouwens et al. (2011). In this plot, we use the dust-corrected $M_{\mathrm{UV}}^{\star}\left(M_{\mathrm{UV}}^{\star}\right.$,cor $)$ rather than observed $M_{\mathrm{UV}}^{\star}$, as the dust-corrected $M_{\mathrm{UV}}^{\star}$ can be used to represent the SFRs in the galaxies. Bouwens et al. $(2009,2012)$ measure the UV-continuum slope, which is a direct indicator of the dust extinction in LBGs from $z \sim 3$ to $z \sim 7$. We adopt their UV slope measurements and the relation between the UV-continuum slope $(\beta)$ and the UV dust extinction ( $A_{\mathrm{UV}}$; Meurer et al. 1999) to correct $M_{\mathrm{UV}}^{\star}$ at different redshifts. For the evolution plot of the SMF, the black triangle is from this work and the two red diamonds at $z \sim 4$ and $z \sim 5$ are from Lee et al. (2012). In 

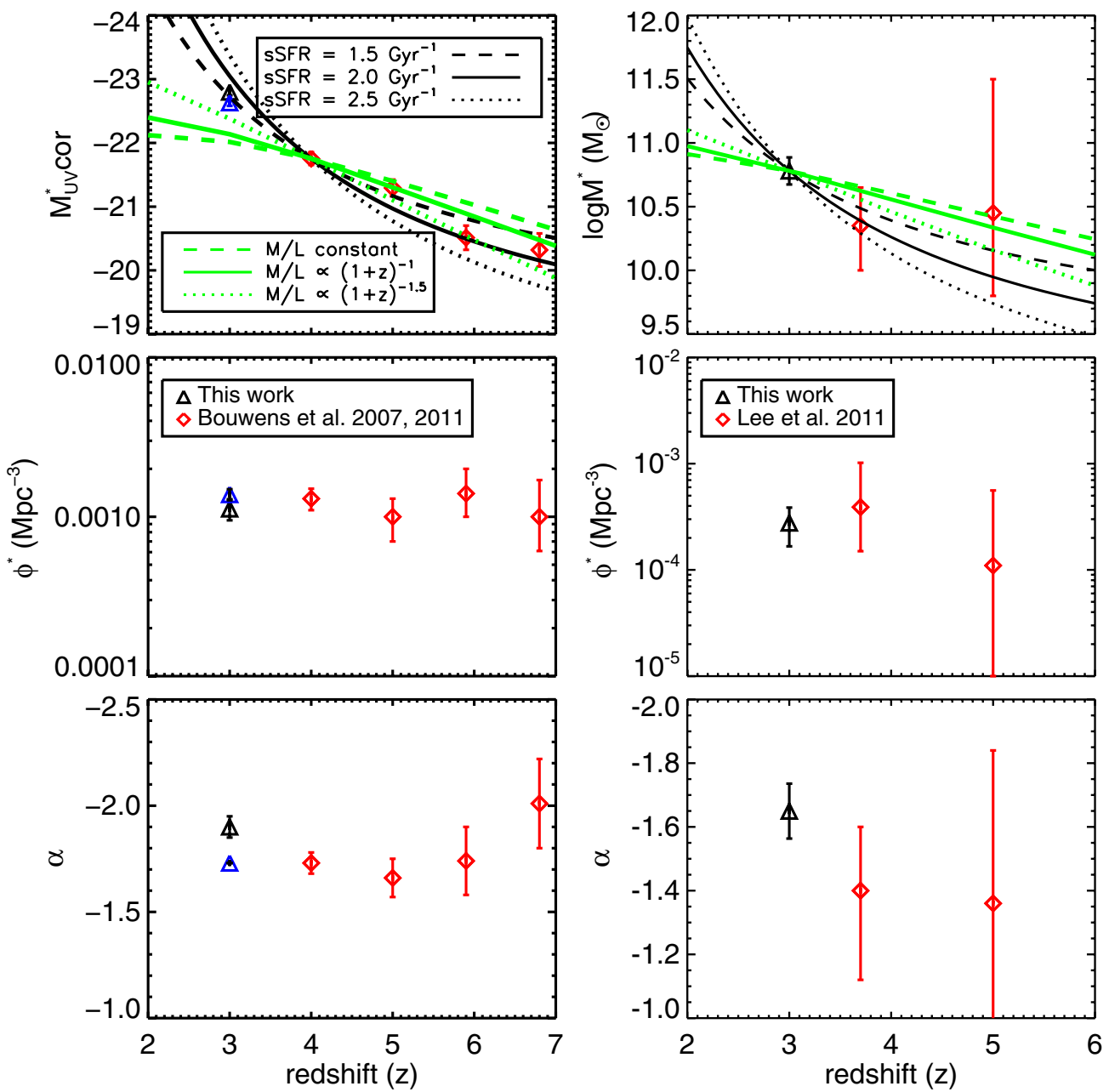

Figure 15. Evolution of best-fit parameters of Schechter functions of UV LF (left panel) and SMF (right panel) with redshifts. Left panel: for characteristic absolute magnitude, we use the dust-corrected value $\left(M_{\mathrm{UV}}^{\star}\right.$, cor). The black triangles are derived by fitting the Schechter function with the combination of LF from this work (bright end) and Reddy \& Steidel (2009, faint end), and the blue ones are derived by fitting the Schechter function with the LF from this work with a fix faint-end slope, $\alpha=1.73$. The red diamonds are from Bouwens et al. (2007, 2011). Right panel: the black triangle is from this work, and the red diamonds are from Lee et al. (2011). The prediction of two different galaxy evolution models are plotted in both panels. The green curves represent the episodic star formation model with different assumptions on evolution of the halo mass to dust-corrected UV luminosity or stellar mass ratio. The green dashed, solid, and dotted curves represent models with the ratio constant with cosmic time, $\propto(1+z)^{-1}$, and $\propto(1+z)^{-1.5}$, respectively. The black solid curves represent the smoothly rising star formation model. The dashed, solid, and dotted black curves represent models with sSFR of $1.5 \mathrm{Gyr}^{-1}, 2.0 \mathrm{Gyr}^{-1}$, and $2.5 \mathrm{Gyr}^{-1}$, respectively.

(A color version of this figure is available in the online journal.)

both the UV LF and SMF, the parameters, $\alpha$ and $\phi^{\star}$, are roughly constant with redshift. On the other hand, the dust-corrected characteristic luminosity, $M_{\mathrm{UV}}^{\star}$, cor, in the UV LF increases with cosmic time. The uncertainty in the characteristic mass, $M_{\text {stellar }}^{\star}$, in the SMF is large in this case, thus it is hard to tell whether or not $M_{\text {stellar }}^{\star}$ increases with cosmic time. The evolutionary trend provides crucial information on how galaxies built up their mass in the early universe. Here we will use two simple toy models to interpret the evolution of the UV LF.

In the first model, we assume that the increasing $M_{\mathrm{UV}}^{\star}$,cor/ $M_{\text {stellar }}^{\star}$ is mainly due to episodic star formation through mergers. In this model, small dark matter halos merge into larger systems and star formation in high- $z$ galaxies is episodic with a duty cycle of $\sim 25 \%$. The increasing $M_{\mathrm{UV}}^{\star} / M_{\text {stellar }}^{\star}$ mainly reflects the increasing mass of host dark matter halos with cosmic time. Bouwens et al. $(2007,2008)$ use this model to interpret the evolution of $M_{\mathrm{UV}}^{\star}$ in the UV LF from $z \sim 6$ to $z \sim 4$. We follow the method in Bouwens et al. (2007) to determine how the halo mass near the knee of the UV LF/SMF increases with time based on the halo mass function of Sheth \& Tormen (1999). We assume three different halo mass to UV luminosity/stellar mass ratio relations: (1) the halo mass to light/stellar mass ratio is constant with cosmic time (green dashed curve-Bouwens et al. 2007); (2) the halo mass to light/stellar mass ratio varies as $(1+z)^{-1}$ (green solid curve-Bouwens et al. 2008); and (3) the halo mass to light/stellar mass ratio varies as $(1+z)^{-1.5}$ (Stark et al. 2007, green dotted curve). The curves are scaled by the $M_{\mathrm{UV}}^{\star}$,cor data point at $z \sim 3$ in the UV LF and by the $M_{\text {stellar }}^{\star}$ data points at $z \sim 4$ in the SMF. The $M_{\mathrm{UV}}^{\star}$,cor $/ M_{\mathrm{stellar}}^{\star}$ in relation (1) increases with time faster than that in relation (2).

In the second model, we assume that (1) $L^{\star} / M_{\text {stellar }}^{\star}$ galaxies are on the main sequence for star-forming galaxies (Daddi et al. 2007), and the specific star formation rate (sSFR $=$ SFR/stellar mass) in LBGs from $z \sim 7$ to $z \sim 3$ is a constant and about 1.5-2.5 $\mathrm{Gyr}^{-1}$ (e.g., Stark et al. 2009; González et al. 2010; Rodighiero et al. 2011); and (2) the LBGs are in a continuous growth stage at this epoch. This model suggests a smoothly rising SFR in the LBGs (e.g., Finlator et al. 2011; Papovich et al. 2011; Lee et al. 2011). The long lasting star formation could be the consequence of cold mode accretion (e.g., Dekel et al. 2009). In this scenario, the LBGs duty cycle is high $(\approx 1)$, and $\phi^{\star}$ does not change with redshift. Therefore, the 
higher redshift $L_{\mathrm{UV}}^{\star} / M_{\text {stellar }}^{\star}$ galaxies are the progenitors of the $L_{\mathrm{UV}}^{\star} / M_{\text {stellar }}^{\star}$ galaxies at lower redshift. As the mass is being built up, $M_{\text {stellar }}^{\star}$ will increase with cosmic time following the relation: $M_{\text {stellar }}^{\star}\left(z_{2}\right)=M_{\text {stellar }}^{\star}\left(z_{1}\right) \times \exp \left(\operatorname{sSFR} \times\left(t\left(z_{2}\right)-t\left(z_{1}\right)\right)\right)$. $L_{\mathrm{UV}}^{*}$ will increase with the same relation. In Figure 15, the black curves are the predicted evolution of $M_{\mathrm{UV}}^{\star} / M_{\text {stellar }}^{\star}$ based on the smoothly rising SFR history model. The dotted, solid, and dashed curves represent different values of the sSFR, which are $1.5 \mathrm{Gyr}^{-1}, 2.0 \mathrm{Gyr}^{-1}$, and $2.5 \mathrm{Gyr}^{-1}$, respectively. The growth rate of $M_{\mathrm{UV}}^{\star} / M_{\text {stellar }}^{\star}$ increases with increasing sSFR. In both models, both $\phi^{\star}$ and $\alpha$ are expected to be constant.

In Figure 15, we compare these two models with our observations. For the evolution of dust-corrected $M_{\mathrm{UV}}^{\star}$, the episodic galaxy merger/star formation history model (green curves) is consistent with the data points between $z \sim 4$ and $z \sim 7$ (Bouwens et al. 2008), while the predicted $M_{\mathrm{UV}}^{\star}$, cor is significantly smaller than the observed $M_{\mathrm{UV}}^{\star}$, cor at $z \sim 3$. On the other hand, the smoothly rising star formation history model (the black curves) can fit the evolution of $M_{\mathrm{UV}}^{\star}$, cor from $z \sim 3$ to $z \sim 7$ very well. For the evolution of the SMF, the predicted $M_{\text {stellar }}^{\star}$ in the episodic star formation model evolves more slowly than that in the smoothly rising star formation history model. The three Schechter parameters are correlated, and the steeper faint-end slope in our LF would result in a higher characteristic luminosity at $z \sim 3$. Therefore, we use the Schechter function with a fixed faint-end slope, $\alpha=1.73$, to fit our LF. The dust-corrected best-fitting characteristic luminosity is shown in Figure 15 (blue triangles). This new LF fitting does not change the characteristic luminosity significantly or our conclusion.

Due to the large uncertainties in the SMF measurements for high-redshift galaxies, especially for galaxies at $z \sim 5$, comparison with models is not conclusive. Larger surveys with smaller statistical errors and cosmic variance are needed to differentiate different models.

Generally speaking, the ratio of UV luminosity to stellar mass, $M_{\mathrm{UV}}^{\star}$, cor $/ M_{\text {stellar }}^{\star}$, evolves much more rapidly in the smoothly rising star formation model than in the episodic star formation model. This is due to the fact that the dark matter halo mass growth rate decreases with cosmic time, while the SFR in galaxies increases with cosmic time from $z \sim 7$ to $z \sim 3$. The halo merger rate is about $1 \mathrm{Gyr}^{-1}$ at $z \sim 5$ for a halo mass of $10^{10} M_{\odot}$ and becomes $0.3 \mathrm{Gyr}^{-1}$ at $z \sim 3$, while the measured sSFR is about $1.5-2.5 \mathrm{Gyr}^{-1}$, which is about a factor of 2-10 larger than the merger rate from $z \sim 5$ to $z \sim 3$. This suggests that the mass build-up in high-redshift galaxies cannot be only from halo merger processes. Other processes, i.e., cold flow accretion, must make a significant contribution to the mass build-up process, especially in the redshift range from $z \sim 5$ to $z \sim 3$. Beyond $z \sim 6$, the merger rate becomes comparable to the sSFR. So at very early epochs $(z>6)$, episodic galaxy assembly could be the dominant process responsible for building up the stellar mass of galaxies.

It is still controversial whether high-redshift LBGs have continuous star formation activity from $z \sim 7$ to $z \sim 3$ (Finlator et al. 2011; Dunlop et al. 2013), or whether the star formation history in LBGs is much shorter with a typical timescale of 300 Myr (Stark et al. 2009; Lee et al. 2011). The relatively young stellar population (Stark et al. 2009) and short star formation duty cycles from clustering measurements (Lee et al. 2009) in high- $z$ LBGs support the latter scenario, in which the star formation history is episodic. On the other hand, Finlator et al. (2011) argue that the short duty cycle from LBG clustering measurements is due to outflow feedback suppression of star formation in low-mass halos, which results in only a small fraction of dark matter halos (0.2-0.4) hosting galaxies. In this scenario, the actual star formation duty cycle is about unity and the star formation history in LBGs is smoothly rising. In summary, our results on the evolution of the UV LF and SMF favor the continuous star formation history model.

\section{CLUSTERING PROPERTIES OF LBGs}

In this section, we measure clustering of the bright LBGs $\left(L>L^{\star}\right)$ at $z \sim 3$. The bright LBG sample is divided into two subsamples with $24.0<R<24.5$ and $23.0<R<24.0$ to study the relation between clustering and LBG luminosity. The clustering properties also allow us to estimate the mass of dark matter halos hosting the LBGs. The real-space three-dimensional (3D) correlation function can be inferred from the two-dimensional (2D) angular correlation function (ACF) by using the redshift distribution information and the Limber function (Limber 1953; Peebles 1980). We do not have the spectroscopic redshifts of individual galaxies, and hence we measure the ACF and model it as $\omega=A_{\omega} \theta^{-\beta}$. Combining the redshift distribution information obtained from our simulation (in Section 5.1), we can obtain the clustering properties, i.e., the comoving correlation lengths for these two subsamples from the ACF.

\subsection{Result}

We use the Landy \& Szalay (1993) estimators to measure the ACF:

$$
\omega(\theta)=\frac{\mathrm{DD}-2 \mathrm{DR}+\mathrm{RR}}{\mathrm{RR}},
$$

where DD, RR, and DR are the numbers of galaxy pairs with a separation between $\theta$ and $\theta+\delta \theta$ in the observed galaxy catalog, the random catalog, and between the observed galaxy and random catalog, respectively. The distribution of the objects in the random catalog for individual fields has exactly the same geometry as that in the galaxy catalog. The objects falling into the areas with $U$-band coverage less than 720 s or without $B_{\mathrm{W}}$ - or $R$-band coverage have been masked out. The number of objects in the random catalog $\left(n_{R}\right)$ is $\sim 100$ times larger than the number of the observed galaxies $\left(n_{G}\right)$.

The Poissonian errors for the ACF are estimated as

$$
\Delta \omega=\sqrt{\frac{1+\omega(\theta)}{\mathrm{DD}}} .
$$

We also calculate the jack-knife errors for the ACF by splitting the whole Boötes field into 63 individual LBC fields, and we find that the jack-knife errors are consistent with the Poissonian errors for the angle separation range from $0.5^{\prime \prime}$ to $200^{\prime \prime}$. This is in agreement with the result of Bielby et al. (2013). Therefore, we adopt the Poissonian errors in the following analysis.

The $\omega(\theta)$ is calculated in each individual LBC pointing field. The final result is the average of the individual $\omega(\theta)$.

The finite survey area makes the clustering results artificially weak. The difference between the true correlation function, $\omega_{\text {true }}(\theta)$, and the measured correlation function, $\omega_{\text {measure }}(\theta)$ is a constant, which is also known as the integral constraint, IC (Adelberger et al. 2005):

$$
\omega_{\text {true }}(\theta)=\omega_{\text {measure }}(\theta)+\mathrm{IC} .
$$

The integral constraint is equal to the matter variance in the size of the survey volume. There are two approaches for estimating 
the integral constraint. One of the methods is to integrate $\omega_{\text {true }}(\theta)$ over the FoV (see details in Roche \& Eales 1999):

$$
\mathrm{IC}=\frac{\sum_{i} \mathrm{RR} \omega\left(\theta_{i}\right)}{\sum_{i} \mathrm{RR}},
$$

and the other method is to use linear theory to estimate the galaxy variance in the survey volume. In this paper, we use the theoretical estimate method to estimate the value of IC (Adelberger et al. 2005; see details in Appendix A). We find the value of IC $\approx 0.02$ for both samples and add it to the measured clustering results, $\omega_{\text {measure }}(\theta)$, to compute the values of $\omega_{\text {true }}(\theta)$.

The ACF is modeled as a power-law form:

$$
\omega(\theta)=A_{\omega} \theta^{-\beta},
$$

with fixed power-law index $\beta=0.6$, which is consistent with the results in Adelberger et al. (2005) and Lee et al. (2006).

The best-fit parameters for the $23.0<R<24.0$ sample are $\left\{A_{\omega}, \beta\right\}=\left\{1.44 \pm 0.14 \operatorname{arcsec}^{\beta}, 0.60\right\}$ and for the $24.0<R<$ 24.5 sample $\left\{A_{\omega}, \beta\right\}=\left\{1.13 \pm 0.06 \operatorname{arcsec}^{\beta}, 0.60\right\}$ (Figure 16).

Then, the $2 \mathrm{D}$ ACF is transformed into the $3 \mathrm{D}$ real space correlation function:

$$
\xi=\left(\frac{r}{r_{0}}\right)^{-\gamma} .
$$

The parameters $r_{0}$ and $\gamma$ can be computed from the following relations:

$$
\begin{aligned}
A= & r_{0}^{\gamma} B[1 / 2,(\gamma-1) / 2] \\
& \times \int_{0}^{\infty} d z N^{2} r(z)^{1-\gamma} g(z)\left[\int_{0}^{\infty} d z N(z)\right]^{-2},
\end{aligned}
$$

(see, e.g., Adelberger et al. 2005; Lee et al. 2006)

$$
\begin{aligned}
& \gamma \equiv \beta+1 \\
& B[1 / 2,(\gamma-1) / 2] \equiv \frac{\sqrt{\pi} \Gamma[(\gamma-1) / 2]}{\Gamma(\gamma / 2)} \\
& g(z) \equiv \frac{H(z)}{c} \\
& r(z)=\int_{0}^{z} \frac{c d z}{H(z)} \\
& H(z)=H_{0} \sqrt{\Omega_{\Lambda}+\Omega_{\mathrm{M}}(1+z)^{3}} .
\end{aligned}
$$

From the above equations, we find that the power-law index $\gamma=1.6$ and the comoving correlation lengths $r_{0}$ for the $23.0<R<24.0$ and $24.0<R<24.5$ LBG samples are $5.77 \pm 0.36 h^{-1} \mathrm{Mpc}$ and $5.14 \pm 0.16 h^{-1} \mathrm{Mpc}$, respectively. The comoving correlation length of the brighter LBG sample is larger than that of the fainter LBG sample at $1 \sigma$ significance level.

The LBGs in this study are about 1-2 mag brighter than those in previous studies. But the survey area and the sample size of the bright LBGs $(R<24.5)$ are an order of magnitude larger, which results in better constraints on the clustering of bright LBGs. In our two bright LBG subsamples, we find that the clustering of LBGs increases with increasing galaxy UV luminosity. This trend has been noted in previous studies of LBGs at different redshifts (Giavalisco et al. 1998; Foucaud et al. 2003; Adelberger et al. 2005; Lee et al. 2006; Hildebrandt et al. 2007, 2009).

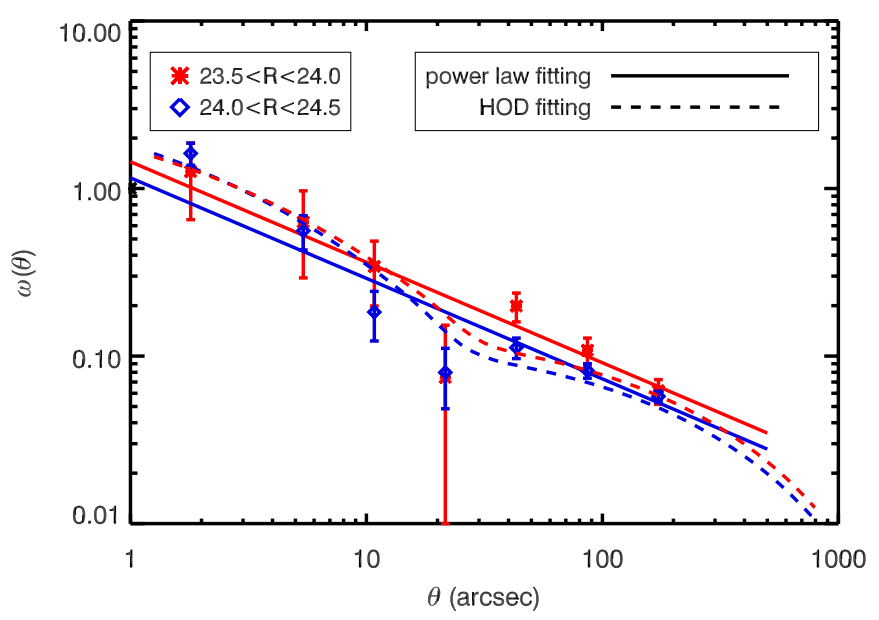

Figure 16. Angular correlation functions for two subsamples at $23.5<R<$ 24.0 (red asterisks) and at $24.0<R<24.5$ (blue diamonds). The red and blue solid lines present the best-fit power law of the bright and faint subsamples, respectively. The red and blue dashed curves are the best-fit HOD models of the bright and faint, respectively.

(A color version of this figure is available in the online journal.)

Lee et al. (2006) and Ouchi et al. (2005) found significant excess power at the small scale of the ACF $\left(\theta<10^{\prime \prime}\right)$ compared to the power-law fit in faint $z \sim 3$ and $z \sim 4$ LBG samples. The excess power is mainly due to the excess number of close galaxy pairs in the same dark matter halos, e.g., the one-halo term. In contrast, we do not find significant excess power at the small scale in our bright LBG sample. This suggests that the one-halo term contribution to the ACF decreases with increasing UV luminosity, and satellite galaxies in dark matter halos are more likely to be faint LBGs rather than bright LBGs.

The correlation lengths in our two subsamples of bright LBGs are significantly larger than those in fainter LBG samples from Adelberger et al. (2005) and Bielby et al. (2011), in which $r_{0} \simeq 4.0 h^{-1}$ Mpc. A similar trend was also found by Foucaud et al. (2003); Lee et al. (2006); Hildebrandt et al. (2007), who also extended the clustering measurements to bright $z \sim 3$ LBGs,

We find that the ACF best-fit parameters in Lee et al. (2006) are similar to those in this study in the similar UV luminosity range. However, our correlation lengths are relatively smaller than those in Lee et al. (2006; Table 3), while Hildebrandt et al. (2007) found similar correlation lengths to those in this work (Table 3). The main reason for this discrepancy is the redshift distribution. Hildebrandt et al. (2009) found that for LBGs at $z \sim 3$ with $r<24.5$ the correlation lengths could vary from 5.0 to $6.0 \mathrm{~h}^{-1} \mathrm{Mpc}$ based on different redshift distributions derived by various methods. The redshift distribution of $z \sim 3$ LBGs in Lee et al. (2006), spanning $z \sim 2$ to $z \sim 4$ (see Figure 1 in Lee et al. 2006), is much broader than that in this study, which can result in a larger correlation length. The redshift distributions from both works are based on simulations, and must be verified by spectroscopic observations.

To obtain the mass of the dark matter halos hosting the bright LBGs, we adopt the HOD models from Hamana et al. (2004) and Lee et al. (2006) and fit them with our ACF measurements (see details in Appendix B). The best-fit HOD models are shown in Figure 16. From the fitting, we can derive the minimum mass of a host halo for the galaxy population, $M_{\min }$, the typical mass of a halo hosting one galaxy, $M_{1}$, and the power-law index, $\alpha$, in Equation (B1), and the best-fit parameters are $\left\{M_{\min }, \alpha, M_{1}\right\}=$ 
Table 3

$\mathrm{ACF}$ and the Comoving Correlation Lengths for $z \sim 3 \mathrm{LBGs}$

\begin{tabular}{lcccc}
\hline \hline Brightness & $A_{\omega}$ & $\beta$ & $r_{0}$ & Ref \\
\hline $23.5<R<24.0$ & $1.44 \pm 0.14$ & 0.6 & $5.77 \pm 0.36$ & This work \\
$24.0<R<24.5$ & $1.13 \pm 0.06$ & 0.6 & $5.14 \pm 0.16$ & This work \\
$R<24.0$ & $1.56_{-0.17}^{+0.14}$ & 0.6 & $7.8_{-0.5}^{+0.4}$ & Lee et al. (2006) \\
$R<24.5$ & $1.16_{-0.08}^{+0.06}$ & 0.6 & $6.5_{-0.3}^{+0.2}$ & Lee et al. (2006) \\
$22.5<24.0$ & $\ldots$ & $0.92 \pm 0.09$ & $6.3 \pm 0.6$ & Hildebrandt et al. (2007) \\
$22.5<24.5$ & $\ldots$ & $0.55 \pm 0.08$ & $5.2 \pm 0.4$ & Hildebrandt et al. (2007)
\end{tabular}

$\left\{8.1_{-1.1}^{+1.4} \times 10^{11} h^{-1} M_{\odot}, 0.5,4.8 \pm 1.0 \times 10^{13} h^{-1} M_{\odot}\right\}$ and $\left\{(1.2 \pm 0.3) \times 10^{12} h^{-1} M_{\odot}, 0.5,7.5_{-2.5}^{+3.0} \times 10^{13} h^{-1} M_{\odot}\right\}$ for the sample of LBGs with $24.0<R<24.5$ and $23.5<R<24.0$, respectively. The mean mass of the host halo can be estimated from:

$$
\left\langle M_{\mathrm{host}}\right\rangle=\frac{\int_{M_{\min }}^{\infty} d M M N_{g}(M) n_{\mathrm{halo}}(M)}{\int_{M_{\min }}^{\infty} d M N_{g}(M) n_{\mathrm{halo}}(M)},
$$

where $n_{\text {halo }}$ is the dark matter halo mass function. The mean masses of the hosting halos for the $24.0<R<24.5$ and $23.5<R<24.0$ samples are $2.5 \pm 0.3 \times 10^{12} h^{-1} M_{\odot}$ and $3.3_{-0.4}^{+0.6} \times 10^{12} h^{-1} M_{\odot}$, respectively. This result confirms that more massive dark matter halos typically host more luminous LBGs, and are consistent with the relation between the UV luminosity and the dark matter halo mass being $L_{\mathrm{UV}} \propto M_{\text {halo }}^{1.5}$. This relation is similar to those for LBGs at $z \sim 4$ and $z \sim 5$ (Ouchi et al. 2005; Lee et al. 2006).

\subsection{Discussion}

We find that LBGs with higher UV luminosities have greater clustering strengths and live in more massive dark matter halos. $R$-band magnitude, which corresponds to the rest-frame UV brightness at redshift $z \sim 3$, is a good tracer for the unobscured star formation in the galaxies. Therefore, this trend also suggests that galaxies with higher SFRs are in more massive dark matter halos. This correlation can be understood in the context of the cold flow mode of galaxy formation: gas is accreted onto dark matter halos from the IGM and finally falls into galaxies within the dark matter halos. This process provides the material to form stars in galaxies. Dekel et al. (2009) derive the corresponding baryonic growth rate in the halo by fitting the growth rate of dark matter halos within the framework of the Extended Press-Schechter formalism (Neistein \& Dekel 2008). They find that the baryonic accretion rate $(\dot{M})$ is a function of halo mass $\left(M_{\text {halo }}\right)$ and redshift $(z)$ :

$$
\dot{M}=6.6\left(\frac{M_{\text {halo }}}{10^{12} M_{\odot}}\right)^{1.15}(1+z)^{2.25} \frac{f_{b}}{0.165} M_{\odot} \mathrm{yr}^{-1},
$$

where $f_{b}=0.165$ is the baryonic fraction in the halos. This relation shows that the cold mode accretion rate increases with increasing redshift and halo mass, suggesting that baryonic accretion is an important process to feed the star formation in high- $z$ star-forming galaxies (Dekel et al. 2009). This model describes how gas is accreted onto dark matter halos at large scales, but does not include any physical processes down to the galaxy scale on how to convert the accreted gas into stars and to regulate star formation. Our measurements of the SFRs in the galaxies and their host dark matter masses will allow us to connect the large scale baryonic accretion process and the small scale star formation process.

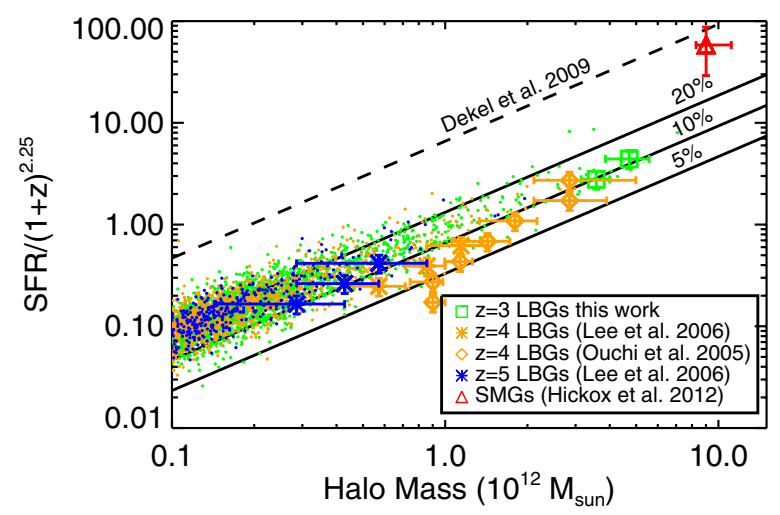

Figure 17. Scaled star formation rate (SFR) vs. hosting halo mass. The dashed line represents the relation in Equation (22) derived from the cold flow model (Dekel et al. 2009), and the solid lines represent the cases where the star formation efficiencies are $20 \%, 10 \%$, and $5 \%$ of the total cold flow accretion mass. The LBGs at $z \sim 3$ (green open squares; this work), $z \sim 4$ (orange asterisks, Lee et al. 2006; orange diamonds, Ouchi et al. 2005), and $z \sim 5$ (blue asterisks; Lee et al. 2006) follow the 10\% cosmic star formation efficiency line very well, while the SMGs (red triangle) at $z \sim 2$ are about 0.85 dex higher. The filled circles are the predictions of the cosmic star formation efficiency from the cosmological simulations with momentum-driven gas outflows recipe (Oppenheimer \& Davé 2008). The points are color coded by redshift (green $z=3$, orange $z=4$, and blue $z=5$ ).

(A color version of this figure is available in the online journal.)

Figure 17 shows the redshift-scaled SFR $\left(\operatorname{SFR} /(1+z)^{2.25}\right)$ as a function of the host halo mass. The SFRs are estimated from dust-corrected UV luminosities. They are derived from the $R$-band magnitude for $z \sim 3$ LBGs in this work, $z$-band magnitude for $z \sim 4$ and 5 LBGs in Lee et al. (2006) and $i$-band magnitude for $z \sim 4$ LBGs in Ouchi et al. (2005), by assuming a typical dust extinction $E(B-V)=0.15$ for LBG samples at $z \sim 3$ and $z \sim 4$ and $E(B-V)=0.10$ for the LBG sample at $z \sim 5$ (e.g., Bouwens et al. 2009). In Figure 17, the dashed line represents the relation of Equation (22), and the three solid lines from top to bottom represent $20 \%, 10 \%$, and $5 \%$ of accreted baryonic gas converted into star formation.

From Figure 17, we find that the observed redshift-scaled SFR as a function of mass from this and other work (Ouchi et al. 2005; Lee et al. 2006) follows the trend predicted by the baryonic accretion model very well over about two orders of magnitude of dark matter halo mass. This relation suggests that the star formation in LBGs is fueled by these baryonic flows and that the cosmic star formation efficiency $(\mathrm{sSFE}=\mathrm{SFR} /(\mathrm{M}))$, which is defined as the efficiency of the conversion of cold flow accretion gas into star formation, is between $5 \%$ and $20 \%$. The cosmic star formation efficiency does not change significantly with redshift $(3<z<5)$, dark halo mass $\left(10^{11}-10^{13} M_{\odot}\right)$, or LBG luminosity $\left(0.1 L^{*}-3 L^{*}\right)$. The low efficiency is consistent with cosmological simulations (e.g., Oppenheimer \& Davé 
2008) in which the cosmic star formation efficiency is about $20 \%$ (Figure 17).

There are two possibilities for the relatively constant cosmic star formation efficiency in a large range of dark matter halo mass. (1) The cosmic star formation efficiency may be set by momentum-driven outflows, which can eject up to $80 \%$ of the total inflow at the smallest halo masses shown, and by retardation of accretion by a hot gaseous halo that emerges at larger halo masses (Kereš et al. 2005). These effects conspire to set the efficiency to about $20 \%$ over this mass range. (2) Dekel $\&$ Birnboim (2006) found that at high redshifts $(z>2)$, narrow cold streams could penetrate directly into the halo, even when the halo mass is above the critical shock-heating mass $M_{\text {shock }} \sim 10^{12}$ (Kereš et al. 2005), therefore, the baryonic accretion efficiency should not change significantly across the critical shock-heating mass, which is in agreement with our relatively constant cosmic star formation efficiency as a function of halo mass. In this case, galaxies with a wide range of luminosities $\left(0.1 L^{*}-3 L^{*}\right)$ should be dominated by a similar feedback process.

The predicted cosmic star formation efficiency from cosmological simulations (e.g., Oppenheimer \& Davé 2008) is about a factor of two higher than the measurements. Therefore, there is probably stronger feedback due to outflows in these galaxies, or other feedback effects need to be introduced to regulate the star formation process. We also note that the systematic uncertainty of the SFR measurements, e.g., different IMFs and dust extinction, can also offset the measured cosmic star formation efficiency by a factor of two or even larger (e.g., Narayanan \& Davé 2012). It is worth noting that although the cosmic star formation efficiency does not change significantly with halo mass, the relation between UV luminosity and halo mass $\left(L \propto M^{1.5}\right)$ in LBGs at $z \sim 3, z \sim 4$, and $z \sim 5$ implies that the cosmic star formation efficiency weakly increases with halo mass.

In Figure 17, we also show the redshift-scaled SFR and the host dark matter halo mass in submillimeter galaxies (SMGs; Hickox et al. 2012). The redshift-scaled SFR in SMGs is about 0.85 dex higher than that in LBGs, suggesting that the intense starburst in the SMGs is not mainly fueled by cold accretion gas but triggered by the major merger process. There are two different star formation modes found, which are long-lasting modes in disk galaxies (e.g., BzK galaxies) fueled by cold flows, and starburst modes in merging galaxies (e.g., SMGs) triggered by major mergers (e.g., Daddi et al. 2010), and the SFRs in starbursts are 4-10 times larger than those in disk galaxies at fixed molecular gas mass. Therefore, the plot demonstrates that LBGs with $L<2.5 L^{*}$ follow a long-lasting mode in disk galaxies, more similar to local spirals and $\mathrm{BzK}$ galaxies.

\section{SUMMARY}

We have carried out an LBC imaging survey in the NDWFS Boötes field, covering the $9 \mathrm{deg}^{2}$ field with $U_{\text {spec }}$ and $Y$-band, to fill in the two main optical wavelength gaps in the NDWFS. The $5 \sigma$ depth of $U_{\text {spec }}$ is $25.5 \mathrm{mag}$. The wide field allows us to select a statistically significant sample of luminous LBGs. Using this sample, we have studied the bright-end LF and clustering properties of LBGs. The main scientific results are summarized as follows.

1. Using the LBT $U_{\text {spec }}$-band images and the NDWFS $B w$ and $R$ images, we selected 14, 485 LBGs at redshift of $\sim 3$ in the $9 \mathrm{deg}^{2}$ NDWFS Boötes field, which is the photometrically selected LBG sample at $z \sim 3$ in the largest area to date.
2. Combined with the faint-end LF measurements from Reddy \& Steidel (2009), we measured the rest-frame UV LF of $z \sim 3$ LBGs. Our large field survey puts a strong constraint on the bright end of the LF. The Schechter function is fit to the UV LF, and the best-fit parameters are $\Phi=(1.06 \pm 0.33) \times 10^{-3} \mathrm{Mpc}^{-3}, M^{\star}=-20.11 \pm 0.08$, and $\alpha=-1.94 \pm 0.10$ by fitting our bright-end data alone, and $\Phi=(1.12 \pm 0.17) \times 10^{-3} \mathrm{Mpc}^{-3}, M^{\star}=-20.08 \pm 0.05$, and $\alpha=-1.90 \pm 0.05$ by combining our bright-end data and the faint-end data points from Reddy \& Steidel (2009). Both results support a steep faint-end slope of the LBG UV LF, rather than a relatively shallower faint-end slope as suggested by Shim et al. (2007) and Sawicki \& Thompson (2006a, 2006b). This implies that the faint LBGs make a significant contribution to the UV LF and dominate the SFR density at $z \sim 3$. With the large survey area and sample of LBGs, this work gives accurate measurement of the bright-end UV LF of $z \sim 3$ LBGs, and allows us to probe the LBG luminosity range $-23.0<M_{\mathrm{UV}}<-25.0$. At the brightest end, the AGN/quasar population dominates the LF. After subtracting the quasar LF from our measured LF, we still found an excess over the Schechter function. Further spectroscopic observations of the galaxy candidates will allow us to confirm the bright LBG LF and study whether the LF follows the Schechter function at the bright end.

3. We estimated the rest-frame near-IR LF of the $z \sim 3$ LBGs. The best-fit Schechter function parameters are $\phi_{J}^{\star}=(3.1 \pm 1.9) \times 10^{-4} \mathrm{Mpc}^{-3}, M_{J}^{\star}=-24.00 \pm 0.30$, and $\alpha_{J}=-2.00 \pm 0.17$. The near-IR LF shows significant evolution compared to that of the rest-frame near-IR of local galaxies. We derived the SMF of the $z \sim 3$ LBGs using the near-IR LF. The density of LBGs is lower than that of $K$-selected galaxies at $z \sim 3$ at the massive end $\left(M>10^{11} M_{\odot}\right)$, suggesting that UV-selected star-forming galaxies do not make a dominant contribution to the SMF of $z \sim 3$ galaxies at the massive end. The LBG SMF shows a steep slope $(\alpha=-1.65 \pm 0.09)$ and dominates the galaxy stellar mass density at the low mass end. By comparing our measured SMF of LBGs with cosmological hydrodynamic simulations with a momentum-driven wind model, we found that the SMF derived from the simulation is consistent with the measured SMF of LBGs at the massive and intermediate mass range, but the simulation overproduces galaxies at the low mass end and does not produce enough massive red and dead galaxies.

4. We studied the evolution of the LBG UV LF and SMF with cosmic time. We compared the evolution with an episodic galaxy formation model and a smoothly rising star formation model, and found that the evolutionary trend supports the model with a steady rising star formation history. In this scenario, galaxies continuously form stars in the redshift range between $z \sim 7$ and $z \sim 3$ and the SFR increases with increasing stellar mass to make the sSFR constant.

5. We also studied the clustering of two samples $(23.5<$ $R<24$ and $24<R<24.5$ ) of the bright LBGs. The comoving correlation lengths, $r_{0}$, for these two samples are found to be $5.77 \pm 0.36 h^{-1} \mathrm{Mpc}$ and $5.14 \pm 0.16 h^{-1} \mathrm{Mpc}$, respectively. This result shows that the galaxies with higher UV luminosity have stronger spatial clustering, implying that the galaxies with higher UV luminosity are hosted by more massive dark matter halos. We used HOD models 
to estimate the mean host dark matter halo mass for these two LBG samples, and found that the mean host halo masses are $2.5 \pm 0.3 \times 10^{12} h^{-1} M_{\odot}$ and $3.3_{-0.4}^{+0.6} \times$ $10^{12} h^{-1} M_{\odot}$, respectively. Combining with other clustering measurements of LBG samples at different redshifts, we found that the relationship of the redshift-scaled SFR and the host halo mass can be interpreted by cold flow gas accreted by the host dark matter halos, and the cosmic star formation efficiency in LBGs is about $5 \%-20 \%$ of the total cold flow gas.

We thank the LBTO staff for great support in preparing the observing and carrying out the observing with LBT/LBC. F.B., X.F., L.J., and I.M. acknowledge support from a Packard Fellowship for Science and Engineering and NSF grants AST 08-06861 and AST 11-07682.

Facility: LBT

\section{APPENDIX A}

\section{INTEGRAL CONSTRAINT}

Following the procedures in Adelberger et al. (2005), we calculate the total IC from:

$$
\mathrm{IC}=\frac{1}{\sum_{1}^{n} \mathrm{RR}_{i}} \sum_{1}^{n} \sigma_{i}^{2} \mathrm{DD}_{i}
$$

where the $\mathrm{RR}_{i}$ is the random pair number in the $i$ th field in the chosen angular bin, i.e., the sum sign means to sum over all the individual field. The $\sigma_{i}$ for each field can be calculated from,

$$
b=\frac{\sigma}{\sigma_{\mathrm{CDM}}}=\frac{\sigma_{8, g}}{\sigma_{8}(z)}
$$

where $\sigma_{8}(z)$ is the linear matter fluctuation in spheres of comoving $8 h^{-1} \mathrm{Mpc}$. We can get it from $\sigma_{8}(z)=\sigma_{8}(0) * D(z)$, where $\sigma_{8}(0)=0.9$, and $D(z)$ is linear growth factor. $\sigma_{8, g}$ is the galaxies variance at the same scale, and it is calculated from

$$
\sigma_{8, g}^{2}=\frac{72\left(r_{0} / 8 h^{-1} \mathrm{Mpc}\right)^{\gamma}}{(3-\gamma)(4-\gamma)(6-\gamma) 2^{\gamma}}
$$

where $r_{0}$ and $\gamma$ are the comoving correlation length and the power-law index in the $3 \mathrm{D}$ correlation function. $\sigma_{\mathrm{CDM}}$, the relative variance of the dark matter from one field to another, can be calculated from

$$
\sigma_{\mathrm{CDM}}=\frac{1}{(2 \pi)^{3 / 2}}\left(\int d^{3} k P_{L}(|k|)\left|W_{k}(k)\right|\right)^{1 / 2},
$$

where $P_{L}(k)$ is the linear cold dark matter (CDM) power spectrum, and $W_{k}$ is the Fourier transform of a survey volume, which can be computed as

$$
W_{k}=\exp \left(\frac{k_{z}^{2} l_{z}^{2}}{2}\right) \frac{\sin \left(k_{x} I_{x} / 2\right)}{k_{x} I_{x} / 2} \frac{\sin \left(k_{y} I_{y} / 2\right)}{k_{y} I_{y} / 2},
$$

where $I_{x}$ and $I_{y}$ are the comoving dimensions of the FoV for each field, and $I_{z}$ is comoving dimensions of the radial direction, which can be convert from the redshift distribution. As the FoV and selection function are almost the same for each field, we adopt the same $\sigma$ for all the fields.

\section{APPENDIX B}

\section{HALO OCCUPATION DISTRIBUTION}

The HOD is applied to our LBG clustering results to interpret host dark matter halo properties for these two bright LBG subsamples. Following the procedure of Hamana et al. (2004), the dark matter halo mass function from Sheth \& Tormen (1999) is used. The number distribution for a given galaxy population as a function of the host dark matter halo is adopted as

$$
N_{g}(M)= \begin{cases}\left(M / M_{1}\right)^{\alpha} & \text { for } M \geqslant M_{\min } \\ 0 & \text { for } M<M_{\min },\end{cases}
$$

where $M_{\min }$ is the minimum mass of a halo hosting the galaxy population, $M_{1}$ is the typical mass of a halo hosting one galaxy, and $\alpha$ is the power-law index. For close galaxy pairs in the same dark matter halo, the following number distribution of the galaxy pairs as a function of halo mass is applied (Bullock et al. 2002):

$$
\left\langle N_{g}\left(N_{g}-1\right)\right\rangle(M)=\left\{\begin{array}{l}
N_{g}^{2}(M) \quad \text { for } N_{g}(M) \geqslant 1 \\
N_{g}^{2}(M) \log \left(4 N_{g}(M)\right) / \log (4) \\
\text { for } 1>N_{g}(M) \geqslant 0.25 \\
0 \quad \text { for } N_{g}(M)<0.25
\end{array}\right.
$$

From the halo mass distribution described above and the galaxy population distribution as a function of halo mass, we can further derive the number density of the galaxy population and the galaxy power spectrum, which is comprised of two components, one is from the galaxy pairs in the same dark matter halo, the one-halo term, and the other is from the galaxies in two different dark matter halos, the two-halo term. Then the galaxy spectrum is converted to the $2 \mathrm{D}$ ACF. We fit both the $2 \mathrm{D}$ ACF and number density of the galaxy population results with our measurements.

\section{REFERENCES}

Adelberger, K. L., Steidel, C. C., Giavalisco, M., et al. 1998, ApJ, 505, 18 Adelberger, K. L., Steidel, C. C., Pettini, M., et al. 2005, ApJ, 619, 697 Arnouts, S., Schiminovich, D., Ilbert, O., et al. 2005, ApJL, 619, L43 Ashby, M. L. N., Stern, D., Brodwin, M., et al. 2009, ApJ, 701, 428 Becker, R. H., White, R. L., \& Helfand, D. J. 1995, ApJ, 450, 559

Bertin, E. 2006, in ASP Conf. Ser. 351, Astronomical Data Analysis Software and Systems XV, ed. C. Gabriel, C. Arviset, D. Ponz, \& S. Enrique (San Francisco, CA: ASP), 112

Bertin, E., \& Arnouts, S. 1996, A\&AS, 117, 393

Bertin, E., Mellier, Y., Radovich, M., et al. 2002, in ASP Conf. Ser. 281, Astronomical Data Analysis Software and Systems XI, ed. D. A. Bohlender, D. Durand, \& T. H. Handley (San Francisco, CA: ASP), 228

Bian, F., Fan, X., Jiang, L., et al. 2012, ApJ, 757, 139

Bielby, R., Hill, M. D., Shanks, T., et al. 2013, MNRAS, 430, 425

Bielby, R. M., Shanks, T., Weilbacher, P. M., et al. 2011, MNRAS, 414, 2

Bouwens, R. J., Illingworth, G. D., Franx, M., et al. 2009, ApJ, 705, 936

Bouwens, R. J., Illingworth, G. D., Franx, M., \& Ford, H. 2007, ApJ, 670, 928

Bouwens, R. J., Illingworth, G. D., Franx, M., \& Ford, H. 2008, ApJ, 686, 230 Bouwens, R. J., Illingworth, G. D., Oesch, P. A., et al. 2011, ApJ, 737, 90

Bouwens, R. J., Illingworth, G. D., Oesch, P. A., et al. 2012, ApJ, 754, 83 Brand, K., Brown, M. J. I., Dey, A., et al. 2006, ApJ, 641, 140

Bruzual, G., \& Charlot, S. 2003, MNRAS, 344, 1000

Bullock, J. S., Wechsler, R. H., \& Somerville, R. S. 2002, MNRAS, 329, 246

Calzetti, D., Armus, L., Bohlin, R. C., et al. 2000, ApJ, 533, 682

Cole, S., Norberg, P., Baugh, C. M., et al. 2001, MNRAS, 326, 255

Cool, R. J. 2007, ApJS, 169, 21

Daddi, E., Dickinson, M., Morrison, G., et al. 2007, ApJ, 670, 156

Daddi, E., Elbaz, D., Walter, F., et al. 2010, ApJL, 714, L118

Davé, R., Oppenheimer, B. D., \& Finlator, K. 2011, MNRAS, 415, 11 de Vries, W. H., Morganti, R., Röttgering, H. J. A., et al. 2002, AJ, 123, 1784 
Dekel, A., \& Birnboim, Y. 2006, MNRAS, 368, 2

Dekel, A., Birnboim, Y., Engel, G., et al. 2009, Natur, 457, 451

Dickinson, M., Papovich, C., Ferguson, H. C., \& Budavári, T. 2003, ApJ, 587,25

Donley, J. L., Rieke, G. H., Pérez-González, P. G., \& Barro, G. 2008, ApJ, 687,111

Dunlop, J. S., Rogers, A. B., McLure, R. J., et al. 2013, MNRAS, 432, 3520

Elston, R. J., Gonzalez, A. H., McKenzie, E., et al. 2006, ApJ, 639, 816

Fan, X., Strauss, M. A., Schneider, D. P., et al. 2001, AJ, 121, 54

Ferguson, H. C., Dickinson, M., Giavalisco, M., et al. 2004, ApJL, 600, L107

Finlator, K., Oppenheimer, B. D., \& Davé, R. 2011, MNRAS, 410, 1703

Foucaud, S., McCracken, H. J., Le Fèvre, O., et al. 2003, A\&A, 409, 835

Franx, M., Labbé, I., Rudnick, G., et al. 2003, ApJL, 587, L79

Giallongo, E., Ragazzoni, R., Grazian, A., et al. 2008, A\&A, 482, 349

Giavalisco, M., Steidel, C. C., Adelberger, K. L., et al. 1998, ApJ, 503, 543

Gonzalez, A. H., Brodwin, M., Brown, M. J. I., et al. 2010, BAAS, 216, 415.13

González, V., Labbé, I., Bouwens, R. J., et al. 2010, ApJ, 713, 115

Hamana, T., Ouchi, M., Shimasaku, K., Kayo, I., \& Suto, Y. 2004, MNRAS, 347,813

Hickox, R. C., Wardlow, J. L., Smail, I., et al. 2012, MNRAS, 421, 284

Hildebrandt, H., Pielorz, J., Erben, T., et al. 2007, A\&A, 462, 865

Hildebrandt, H., Pielorz, J., Erben, T., et al. 2009, A\&A, 498, 725

Hoopes, C. G., Heckman, T. M., Jannuzi, B. T., et al. 2003, BAAS, 35, 1371

Hunt, M. P., Steidel, C. C., Adelberger, K. L., \& Shapley, A. E. 2004, ApJ, 605,625

Isobe, T., Feigelson, E. D., \& Nelson, P. I. 1986, ApJ, 306, 490

Jain, B., \& Lima, M. 2011, MNRAS, 411, 2113

Jannuzi, B. T., \& Dey, A. 1999, in ASP Conf. Ser. 191, Photometric Redshifts and the Detection of High Redshift Galaxies, ed. R. Weymann, L. StorrieLombardi, M. Sawicki, \& R. Brunner (San Francisco, CA: ASP), 111

Kajisawa, M., Ichikawa, T., Tanaka, I., et al. 2009, ApJ, 702, 1393

Kenter, A., Murray, S. S., Forman, W. R., et al. 2005, ApJS, 161, 9

Kereš, D., Katz, N., Weinberg, D. H., \& Davé, R. 2005, MNRAS, 363, 2

Kochanek, C. S., Eisenstein, D. J., Cool, R. J., et al. 2012, ApJS, 200, 8

Kroupa, P. 2001, MNRAS, 322, 231

Lacey, C. G., Baugh, C. M., Frenk, C. S., \& Benson, A. J. 2011, MNRAS, 412, 1828

Lacy, M., Storrie-Lombardi, L. J., Sajina, A., et al. 2004, ApJS, 154, 166

Landy, S. D., \& Szalay, A. S. 1993, ApJ, 412, 64

Le Fèvre, O., Paltani, S., Arnouts, S., et al. 2005, Natur, 437, 519

Lee, K.-S., Dey, A., Reddy, N., et al. 2011, ApJ, 733, 99

Lee, K.-S., Ferguson, H. C., Wiklind, T., et al. 2012, ApJ, 752, 66

Lee, K.-S., Giavalisco, M., Conroy, C., et al. 2009, ApJ, 695, 368

Lee, K.-S., Giavalisco, M., Gnedin, O. Y., et al. 2006, ApJ, 642, 63

Limber, D. N. 1953, ApJ, 117, 134

Ly, C., Malkan, M. A., Treu, T., et al. 2009, ApJ, 697, 1410

Madau, P. 1995, ApJ, 441, 18

Madau, P., Ferguson, H. C., Dickinson, M. E., et al. 1996, MNRAS, 283, 1388

Marchesini, D., van Dokkum, P. G., Förster Schreiber, N. M., et al. 2009, ApJ, 701,1765
Meurer, G. R., Heckman, T. M., \& Calzetti, D. 1999, ApJ, 521, 64

Mo, H. J., \& White, S. D. M. 1996, MNRAS, 282, 347

Murray, N., Quataert, E., \& Thompson, T. A. 2005a, ApJ, 618, 569

Murray, S. S., Kenter, A., Forman, W. R., et al. 2005b, ApJS, 161, 1

Narayanan, D., \& Davé, R. 2012, MNRAS, 423, 3601

Neistein, E., \& Dekel, A. 2008, MNRAS, 388, 1792

Oke, J. B., \& Gunn, J. E. 1983, ApJ, 266, 713

Oppenheimer, B. D., \& Davé, R. 2008, MNRAS, 387, 577

Oppenheimer, B. D., Davé, R., Kereš, D., et al. 2010, MNRAS, 406, 2325

Ouchi, M., Hamana, T., Shimasaku, K., et al. 2005, ApJL, 635, L117

Papovich, C., Finkelstein, S. L., Ferguson, H. C., Lotz, J. M., \& Giavalisco, M. 2011, MNRAS, 412, 1123

Peebles, P. J. E. 1980, The Large-scale Structure of the Universe (Princeton: Princeton Univ. Press)

Poli, F., Menci, N., Giallongo, E., et al. 2001, ApJL, 551, L45

Reddy, N. A., Pettini, M., Steidel, C. C., et al. 2012, ApJ, 754, 25

Reddy, N. A., \& Steidel, C. C. 2009, ApJ, 692, 778

Reddy, N. A., Steidel, C. C., Pettini, M., et al. 2008, ApJS, 175, 48

Roche, N., \& Eales, S. A. 1999, MNRAS, 307, 703

Rodighiero, G., Daddi, E., Baronchelli, I., et al. 2011, ApJL, 739, L40

Salpeter, E. E. 1955, ApJ, 121, 161

Sawicki, M., \& Thompson, D. 2006a, ApJ, 642, 653

Sawicki, M., \& Thompson, D. 2006b, ApJ, 648, 299

Schmidt, M. 1968, ApJ, 151, 393

Schmitt, J. H. M. M. 1985, ApJ, 293, 178

Scoville, N., Aussel, H., Brusa, M., et al. 2007, ApJS, 172, 1

Shapley, A. E., Steidel, C. C., Adelberger, K. L., et al. 2001, ApJ, 562, 95

Sheth, R. K., \& Tormen, G. 1999, MNRAS, 308, 119

Shim, H., Im, M., Choi, P., Yan, L., \& Storrie-Lombardi, L. 2007, ApJ, 669,749

Soifer, B. T., \& Spitzer/NOAO Team. 2004, BAAS, 36, 746

Spergel, D. N., Bean, R., Doré, O., et al. 2007, ApJS, 170, 377

Stark, D. P., Ellis, R. S., Bunker, A., et al. 2009, ApJ, 697, 1493

Stark, D. P., Loeb, A., \& Ellis, R. S. 2007, ApJ, 668, 627

Steidel, C. C., Adelberger, K. L., Giavalisco, M., Dickinson, M., \& Pettini, M. 1999, ApJ, 519, 1

Steidel, C. C., Adelberger, K. L., Shapley, A. E., et al. 2003, ApJ, 592, 728

Steidel, C. C., Giavalisco, M., Dickinson, M., \& Adelberger, K. L. 1996a, AJ, 112,352

Steidel, C. C., Giavalisco, M., Pettini, M., Dickinson, M., \& Adelberger, K. L. 1996b, ApJL, 462, L17

Steidel, C. C., Shapley, A. E., Pettini, M., et al. 2004, ApJ, 604, 534

Stern, D., Eisenhardt, P., Gorjian, V., et al. 2005, ApJ, 631, 163

Trenti, M., \& Stiavelli, M. 2008, ApJ, 676, 767

van der Burg, R. F. J., Hildebrandt, H., \& Erben, T. 2010, A\&A, 523, A74

van Dokkum, P. G. 2001, PASP, 113, 1420

van Dokkum, P. G., Quadri, R., Marchesini, D., et al. 2006, ApJL, 638, L59

Yan, H., Finkelstein, S. L., Huang, K.-H., et al. 2012, ApJL, 761, L77

Yan, H., Yan, L., Zamojski, M. A., et al. 2011, ApJL, 728, L22

Zheng, Z., Coil, A. L., \& Zehavi, I. 2007, ApJ, 667, 760 